\title{
Endovascular Recanalization Therapy in Acute Ischemic Stroke: Updated Meta-analysis of Randomized Controlled Trials
}

\author{
Keun-Sik Hong, ${ }^{\text {a* }}$ Sang-Bae Ko, ${ }^{\text {b* }}$ Ji Sung Lee, ${ }^{\mathrm{c}}$ Kyung-Ho Yu, ${ }^{\mathrm{d}}$ Joung-Ho Rha ${ }^{\mathrm{e}}$ \\ ${ }^{a}$ Department of Neurology, Inje University Ilsan Paik Hospital, Goyang, Korea \\ ${ }^{b}$ Department of Neurology, Seoul National University College of Medicine, Seoul, Korea \\ ${ }^{\mathrm{c}}$ Clinical Research Center, Asan Medical Center, Seoul, Korea \\ ${ }^{d}$ Department of Neurology, Hallym University College of Medicine, Anyang, Korea \\ eDepartment of Neurology, Inha University College of Medicine, Incheon, Korea
}

Background and Purpose Recent randomized clinical trials (RCTs) have demonstrated benefits of endovascular recanalization therapy (ERT) contrary to earlier trials. We aimed to estimate the benefits of ERT added to standard therapy in acute ischemic stroke.

Methods From a literature search of RCTs testing ERT, we performed a meta-analysis to estimate an overall efficacy and safety of ERT for all trials, stent-retriever trials, and RCTs comparing ERT and intravenous tissue plasminogen activator (IV-TPA).

Results We identified 15 relevant RCTs including 2,899 patients. For all trials, ERT was associated with increased good outcomes (odds ratio [OR] 1.79; 95\% confidence interval [Cl] $1.34,2.40 ; P<0.001)$ compared to the control. ERT also increased no or minimal disability outcomes, good neurological recovery, good activity of daily living, and recanalization. ERT did not significantly increase symptomatic intracranial hemorrhage (SICH) (OR 1.19; $95 \% \mathrm{Cl}$ $0.83,1.69 ; P=0.345)$ or death (OR $0.87 ; 95 \% \mathrm{Cl} 0.71,1.05 ; P=0.151)$. In contrast, ERT significantly reduced extreme disability or death (OR $0.77 ; 95 \% \mathrm{Cl} 0.61,0.97 ; P=0.025)$. Restricting to five stent-retriever trials comparing ERT plus IV-TPA vs. IV-TPA alone, the benefit was even greater for good outcome (OR 2.39; $95 \% \mathrm{Cl} 1.88,3.04 ; P<0.001)$ and extreme disability or death (OR 0.57; 95\% $\mathrm{Cl} 0.41,0.78 ; P=0.001)$. Restricting to eight RCTs comparing ERT (plus IV-TPA in six trials) with IV-TPA alone showed similar efficacy and safety.

Conclusions This updated meta-analysis shows that ERT substantially improves clinical outcomes and reduces extreme disability or death without significantly increasing $\mathrm{SICH}$ compared to standard therapy.

Keywords Acute ischemic stroke; Intra-arterial; Thrombolysis; Thrombectomy; Meta-analysis

\author{
Correspondence: Keun-Sik Hong \\ Department of Neurology, Stroke Center, \\ Ilsan Paik Hospital, Inje University, 170 \\ Juhwa-ro, Ilsanseo-gu, Goyang 10380, \\ Korea \\ Tel: $+82-31-910-7680$ \\ Fax: +82-31-910-7368 \\ E-mail:nrhks@paik.ac.kr
}

Co-correspondence: Joung-Ho Rha Department of Neurology, Inha University Hospital, Inha University College of Medicine, 27 Inhang-ro, Jung-gu, Incheon 22332, Korea Tel: +82-32-890-3668 Fax: +82-32-890-3864 E-mail:jhrha@inha.ac.kr

*Keun-Sik Hong and Sang-Bae Ko contributed equally to the manuscript as first authors.

Received: May 29, 2015

Revised: July 16, 2015

Accepted: July 16, 2015

The authors have no financial conflicts of interest.

\section{Introduction}

Intravenous tissue plasminogen activator (IV-TPA) administered within 4.5 hours after stroke onset is the only proven therapy for improving outcomes in patients with acute ischemic stroke (AIS). ${ }^{1,2}$ However, acute large artery occlusions are usually resistant to IV-TPA. ${ }^{3,4}$ Endovascular recanalization therapy (ERT) has an advantage of a higher recanalization rate, but disadvantages of longer time required for treatment initiation, the need for advanced and resource-intensive stroke care system, 
and potential increase in symptomatic intracranial hemorrhage $(\mathrm{SICH})$.

In an earlier meta-analysis of randomized controlled trials (RCTs), ERT substantially increased recanalization rates and improved clinical outcomes in AIS compared to no thrombolytic therapy. Despite an increased risk of SICH, ERT did not increase mortality. ${ }^{5}$ However, two RCTs failed to demonstrate any benefit of ERT (used alone or in addition to IV-TPA) compared to IV-TPA alone. ${ }^{4,6}$ However, recent RCTs using stent-retriever thrombectomy have consecutively demonstrated the benefits of ERT plus IV-TPA over IV-TPA alone. ${ }^{7-11}$

We conducted a meta-analysis of all published RCTs to generate an overall estimate of the benefits of ERT when added to standard therapy.

\section{Methods}

We systematically searched PubMed and EMBASE databases (January 1998 to May 2015) with the search terms "ischemic stroke” AND "intra-arterial” AND "thrombolysis or thrombectomy." We restricted our search to human and clinical trials and included no language restrictions. In addition, we performed manual searches of the bibliographies of all trials. Abstracts and articles were reviewed and identified by two investigators (Ko $\mathrm{SB}$ and Hong KS). Inclusion criteria were 1) RCT, 2) active arm receiving ERT, 3) control arm receiving standard therapy including IV-TPA but not ERT, and 4) modified Rankin Scale (mRS) score reported at 90 days or at the end of the trial. We assessed study quality using the Cochrane risk-of-bias algorithm (www. cochrane.org/training/cochrane-handbook). ${ }^{12}$

All data from selected RCTs were independently abstracted by two investigators (Ko SB and Hong KS). The quality of each study was also reviewed by another two investigators (Yu KH and $\mathrm{Rha} \mathrm{JH}$ ). Discrepancies were resolved by consensus and by referencing the original reports. In most outcomes, analyses were based on the intention-to-treat population (SICH rate on perprotocol population in one trial). ${ }^{13}$ The Cochrane Collaboration's Review Manager Software Package (RevMan 5) was used for this meta-analysis. Using a random-effect model, we generated a pooled estimate as an odds ratio (OR) with a $95 \%$ confidence interval (CI). All statistical tests were 2 -sided, and a $P$ value of $<0.05$ was considered statistically significant.

Primary efficacy outcome was good outcome defined by an $\mathrm{mRS}$ score of $0-2$. Secondary efficacy outcomes were mRS 0-1, mRS 0-3, good neurological outcome (variable criteria on the National Institutes of Health Stroke Scale [NIHSS] score across trials), good activity of daily living (Barthel Index 85, 90, or 95 to 100); partial or complete recanalization modified arterial occlu- sive lesion (mAOL) recanalization score, Thrombolysis in Myocardial Infarction (TIMI) grade, Thrombolysis in Cerebral Infarction (TICI) 2-3, and complete recanalization (mAOL, TIMI, or TICI 3). Safety outcomes were SICH, any ICH including asymptomatic $\mathrm{ICH}$ and $\mathrm{SICH}$, bed-ridden status or death (mRS 5-6), and mortality. We compared outcomes between the ERT and control groups for all RCTs, stent-retriever trials (stentretriever thrombectomy $>75 \%$ of ERT), and RCTs of control arms with IV-TPA therapy (IV-TPA rate > 75\%). Using data from five stent-retriever trials, shift analysis (shift in disability levels on $\mathrm{mRS}$ ) was conducted to analyze the effect of ERT on the distribution of mRS outcomes. Heterogeneity was assessed by $P$ values of $\chi^{2}$ statistics and by $\mathrm{I}^{2}$ statistics. Heterogeneity was considered significant if the $P$ values of $\chi^{2}$ statistics were $<0.10$. We regarded an $\mathrm{I}^{2}$ of $<40 \%$ as minimal heterogeneity, $40 \%-75 \%$ as modest heterogeneity, and $>75 \%$ as substantial heterogeneity. ${ }^{12}$ Publication bias was assessed graphically with a funnel plot, and statistically with the Begg's test.

\section{Results}

\section{Study selection and characteristics}

From our review of abstracts and full articles identified through the literature search, our final analysis included 15 RCTs including 2,899 patients: 1,575 patients were randomized to ERT arms and 1,324 to control arms (Figure 1). ${ }^{4,6-11,13-20}$ The characteristics of the included trials are presented in Table 1. In brief, mean age ranged between 58.7 and 71.5 years, median NIHSS score between 13 and 26, and time window between 3.0 and 24 hours (6 hours in eight RCTs). Before randomization, large artery occlusion was confirmed in all patients in $10 \mathrm{RCTs}, 43.0 \%$ in one $\mathrm{RCT}^{4}$ and none in four RCTs. ${ }^{6,15,16,19}$ Of the 15 RCTs, 11 exclusively enrolled patients with anterior circulation stroke, and only one RCT exclusively enrolled patients with posterior circulation stroke. In three RCTs, most patients had anterior circulation strokes, and 3.0\%-19.3\% of patients had posterior circulation strokes (Table 1). The assessment of the risk of bias in each trial is presented in Figure 2. In general, most RCTs were at a low risk of bias for each item except for blinding of treatment assignment.

In nine trials, the control arms received IV-TPA (IV-TPA rates between $29.6 \%^{20}$ and $100 \%{ }^{4,9,10}$ ), whereas, in six trials, ${ }^{13-18}$ no patient received IV-TPA (intravenous urokinase in one trial). ${ }^{16}$ In eight RCTs, ${ }^{4,6-11,19}$ the IV-TPA rate in the control arm was greater than $75 \%$. For the ERT arms, the actual rates of providing ERT ranged between $76.0 \%$ and $100 \%$, and five RCTs mainly used stent-retriever (ranged from $77.1 \%$ to $95.1 \%$ ). ${ }^{7-11}$ The disability outcome measured by mRS score was assessed at 90 days in 13 RCTs, 30 days in one, ${ }^{15}$ and 180 days in one. ${ }^{17}$ 


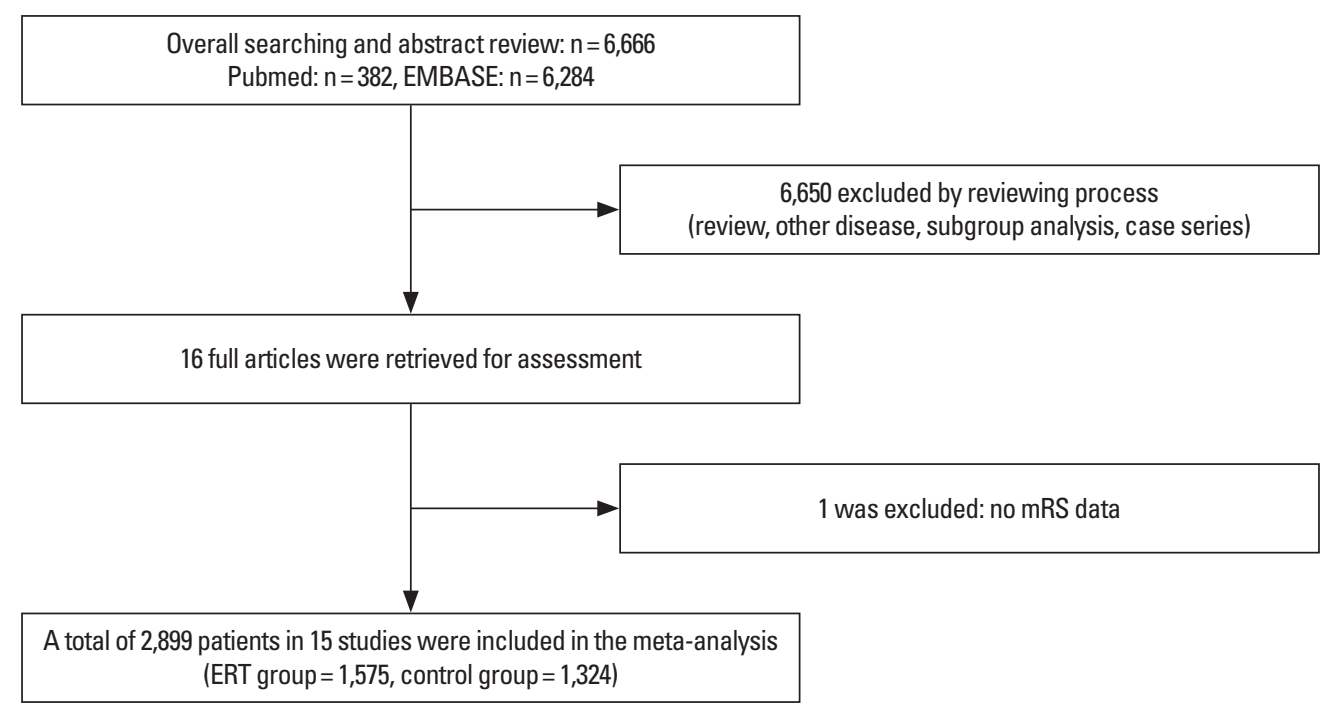

Figure 1. Selection of studies for inclusion in the meta-analysis.

\section{Primary efficacy outcome: mRS 0-2}

Pooling of the results of 13 RCTs comparing ERT versus the control (1,517 in ERT arms vs. 1,261 in control arms) from the random-effect model showed that ERT was associated with increased mRS 0-2 outcome (43.3\% vs. $31.9 \%$; OR [95\% CI], $1.79[1.34,2.40] ; P<0.001$; number needed-to-treat $[\mathrm{NNT}]=$ 9). When restricted to five stent-retriever RCTs (633 in ERT arms vs. 645 in control arms), ERT was associated with increased mRS 0-2 outcome (46.1\% vs. 26.4\%; 2.39 [1.88, 3.04]; $P<0.001$; NNT $=5)$. When analyzing eight RCTs with IV-TPA control arms (IV-TPA rate > 75\%) (1,254 in ERT arms vs. 1,069 in control arms), the ERT group was more likely to achieve mRS 0 -2 outcome (44.6\% vs. $32.6 \% ; 1.88[1.29,2.73]$; $P=0.001$; NNT $=8$ ) (Tables 2-4 and Figure 3).

\section{Secondary efficacy outcomes}

Pooled estimates for secondary efficacy outcomes are presented in Tables 2-4 and Figures 4 and 5 (supplementary Figures 1-4). ERT was associated with higher frequencies of $\mathrm{mRS} 0-1$, mRS 0-3, good neurological outcome, good activity of daily living, partial or complete recanalization (mAOL, TIMI, or TICI 2-3), and complete recanalization in all RCTs, stent-retriever trials, and RCTs of control arms with IV-TPA compared to control. In five stent-retriever trials, ERT was significantly associated with a favorable shift in $\mathrm{mRS}$ score distribution (indicating the odds of improving one or more score on the $\mathrm{mRS})(2.02[1.64,2.48]$; $P<0.001$ ) (Table 3).

\section{Safety outcomes}

Pooled estimates for safety outcomes are presented in Tables
2-4 and Figures 6-8. When pooling all 15 RCTs (1,562 in ERT arms vs. 1,318 in control arms), SICH was more frequent with ERT compared to the control, but the difference was not statistically significant ( $5.8 \%$ vs. $4.6 \% ; 1.19[0.83,1.69]$; $P=0.345$; number needed-to-harm $=88$ ). The SICH rate was almost identical between the ERT and control groups in five stent-retriever trials ( $4.1 \%$ vs. $4.3 \% ; 1.08[0.61,1.88] ; P=0.798)$ and eight RCTs with control arms with IV-TPA (5.1\% vs. 5.1\%; 1.03 [0.70, 1.50]; $P=0.893)$. Any ICH was significantly increased with ERT in all trials and RCTs with control arms with IV-TPA, but was not significantly increased with stent-retriever thrombectomy (Supplementary Figure 5).

Mortality rate was non-significantly lower in the ERT group than in the control group in all 15 RCTs (17.6\% vs. $19.4 \%$; 0.87 $[0.71,1.05] ; P=0.151 ; \mathrm{NNT}=55)$, five stent-retriever trials (15.3\% vs. $18.8 \% ; 0.78[0.54,1.12] ; P=0.1770 ; \mathrm{NNT}=29)$, and eight RCTs with $>75 \%$ IV-TPA rates in their control arms $(16.9 \%$ vs. $18.0 \% ; 0.90[0.69,1.17] ; P=0.4317$; NNT =93). However, ERT compared to control significantly reduced the extreme disability or death outcome (mRS 5-6) in all RCTs (12 RCTs: $24.3 \%$ vs. $29.2 \%$; $0.77[0.61,0.97] ; P=0.025 ; \mathrm{NNT}=21)$, five stent-retriever trials ( $21.6 \%$ vs. $32.2 \%$; 0.57 [0.41, 0.78$]$; $P=0.001$; NNT =9), and eight RCTs with control arms with IV-TPA (22.5\% vs. $28.7 \%$; $0.70[0.52,0.93] ; P=0.013$; NNT $=16$ ).

\section{Publication bias}

No publication bias was found for all outcomes except for SICH in stent-retriever RCTs (Table 2). However, for the SICH outcome, it was likely caused by the extremely low rate of SICH with a wide confidence interval in two trials. ${ }^{9,10}$ 
Table 1. Characteristics of Included Trials

\begin{tabular}{|c|c|c|c|c|c|c|c|c|c|}
\hline \multirow{2}{*}{$\begin{array}{l}\text { Trial } \\
\text { Publication year }\end{array}$} & \multicolumn{2}{|c|}{ PROACT $^{14}$} & PROACT $\|^{13}$ & \multicolumn{2}{|c|}{ Keris et al. ${ }^{15}$} & Ducrocq et al. ${ }^{16}$ & Macleod et al. ${ }^{17}$ & MELT $^{18}$ & SYNTHESIS pilot ${ }^{19}$ \\
\hline & & 1998 & 1999 & \multicolumn{2}{|c|}{2001} & 2005 & 2005 & 2007 & 2010 \\
\hline Countries & & USA, Canada & USA, Canada & \multicolumn{2}{|c|}{ Latvia } & France & Australia & Japan & Italy \\
\hline Participants (n) & & 40 & 180 & \multicolumn{2}{|c|}{45} & 27 & 16 & 114 & 54 \\
\hline Age (year) & \multicolumn{2}{|c|}{67.6} & 64.0 & \multicolumn{2}{|c|}{61.8} & 58.7 & 63.9 & 67.1 & 62.4 \\
\hline Female $(\%)$ & \multicolumn{2}{|c|}{52.5} & 41.1 & \multicolumn{2}{|c|}{40.0} & 25.9 & 37.5 & 35.1 & 22.2 \\
\hline Baseline NIHSS (ERT/control) & & 7/19 & $17 / 17$ & & $25 / 26$ & NA & 23/18 & $14 / 14$ & $17 / 16$ \\
\hline Time window (hour) & & 6.0 & 6.0 & & 6.0 & 6.0 & 24.0 & 6.0 & 3.0 \\
\hline Anterior circulation stroke (\%) & & 00.0 & 100.0 & & 100.0 & 100.0 & 0 & 100.0 & 80.7 \\
\hline $\begin{array}{l}\text { LAO confirmation before randomiza } \\
\text { (confirmation, \%) }\end{array}$ & ation & (100.0) & Yes (100.0) & $\begin{array}{r}\text { No }(L A O \\
\mathrm{pa}\end{array}$ & $\begin{array}{l}0 \text { in all ERT } \\
\text { atients) }\end{array}$ & $\begin{array}{l}\text { No (LAO in all ERT } \\
\text { patients) }\end{array}$ & Yes (100.0) & Yes (100.0) & No (NA) \\
\hline ICA occlusion $(\%)$ & & 2.5 & NA & 25.0 (ERT & RT arm only) & 7.4 (ERT arm only) & BA or VA occlusior & 0 & NA \\
\hline M1 occlusion (\%) & & 2.5 & 61.7 & 41.7 (ERT & RT arm only) & 40.7 (ERT arm only) & BA or VA occlusiol & 71.0 & NA \\
\hline Infarct core or penumbral selection & & lone & None & & Vone & $\begin{array}{l}\text { CT hypointensity } \\
<1 / 3 \text { MCA territory }\end{array}$ & None & None & None \\
\hline $\begin{array}{l}\text { Onset to randomization/groin punct } \\
\text { first reperfusion (minute) }\end{array}$ & ture/ & $276 / 330$ & 90/NA/318 & $\mathrm{NA} / \mathrm{I}$ & $\mathrm{NA} / 229$ & NA/NA/324 & NA/NA/710 & 197/NA/227 & 125/NA/195 \\
\hline ERT arm & IAp & ro-UK & IA pro-UK & TPA & $(I A+I V)$ & IAUK & IAUK & IAUK & IA only \\
\hline Control arm & & acebo & placebo & & hone & IV UK & none & none & IV-TPA \\
\hline IV-TPA (active/control) (\%) & & $0 / 0$ & $0 / 0$ & & $00 / 0$ & $0 / 0$ & $0 / 0$ & $0 / 0$ & $0 / 96.6$ \\
\hline ERT performed in ERT arm (\%) & & 00.0 & 89.3 & & 00.0 & 100.0 & 100.0 & 98.2 & 76.0 \\
\hline Stent-Retriever in active arm (\%) & & 0.0 & 0.0 & & 0.0 & 0.0 & 0.0 & 0.0 & 4.0 \\
\hline mTICI 2b-3 in active arm (\%) & & NA & NA & & NA & 31 & NA & 53.0 & NA \\
\hline Outcome assessment (day) & & 90 & 90 & & 30 & 90 & 180 & 90 & 90 \\
\hline Trial & $\begin{array}{l}\text { SYNTHESIS } \\
\text { EXPANSION }\end{array}$ & MRRESCUE ${ }^{20}$ & & & MR CLEAN ${ }^{\top}$ & ESCAPE $^{8}$ & EXTEND-IA ${ }^{9}$ & SWIFT PRIME ${ }^{10}$ & REVASCAT $^{11}$ \\
\hline Publication year & 2013 & 2013 & & & 2014 & 2015 & 2015 & 2015 & 2015 \\
\hline Countries & Italy & North America & Intern & ational & Netherlands & International & $\begin{array}{l}\text { Australia and } \\
\text { New Zealand }\end{array}$ & USA and Europe & Spain \\
\hline Participants (n) & 362 & 118 & & 56 & 500 & 315 & 70 & 196 & 206 \\
\hline Age (year) & 66.5 & 65.5 & & 7 & 65.7 & 71.5 & 69.4 & 65.6 & 66.5 \\
\hline Female (\%) & 42.3 & 51.7 & & 2 & 41.6 & 52.4 & 51.0 & 49.0 & 47.1 \\
\hline Baseline NIHSS (active/control) & $13 / 13$ & $17.4 / 17.7$ & & 16 & $17 / 18$ & $16 / 17$ & $17 / 13$ & $17 / 17$ & $17 / 17$ \\
\hline Time window (hour) & 4.5 & 8.0 & & 0 & 6.0 & 12.0 & 6.0 & 6.0 & 8.0 \\
\hline Anterior circulation stroke (\%) & 92.0 & 100.0 & & 0 & 100.0 & 100.0 & 100.0 & 100.0 & 100.0 \\
\hline $\begin{array}{l}\text { LAO confirmation before } \\
\text { randomization (confirmation, \%) }\end{array}$ & No (NA) & Yes (100.0) & No & 43.0) & Yes (100.0) & Yes (100.0) & Yes (100.0) & Yes (100.0) & Yes (100.0) \\
\hline ICA occlusion (\%) & NA & 16.9 & 22.1 (ERT & arm only) & 27.6 & 26.7 & 31.4 & 16.3 & 26.2 \\
\hline M1 occlusion (\%) & NA & 66.1 & 41.1 (ERT & arm only) & 63.8 & 68.6 & 54.3 & 68.4 & 63.6 \\
\hline $\begin{array}{l}\text { Infarct core or penumbral } \\
\text { selection }\end{array}$ & None & $\begin{array}{l}\text { Infarct core } \\
\leq 90 \mathrm{~mL} \text { on CT } \\
\text { or MRI }\end{array}$ & $\begin{array}{r}\text { CT hypo } \\
\text { ' } 1 / 3 \mathrm{MC} \\
\text { or ASPE }\end{array}$ & $\begin{array}{l}\text { intensity } \\
\text { A territory } \\
\text { CTS } \geq 4\end{array}$ & None & $\begin{array}{c}\text { ASPECTS } \geq 6 \text { and } \\
\text { moderate-to-good } \\
\text { collaterals }\end{array}$ & $\begin{array}{c}\quad \text { Infarct core } \\
\leq 70 \mathrm{~mL} \text { and Mis- } \\
\text { match on CT/MRI }\end{array}$ & $\begin{array}{c}\text { CT/MRI } \\
\text { ASPECTS } \geq 6^{*}\end{array}$ & $\begin{array}{c}\text { CT ASPECTS } \geq 7 \\
\text { or DWI AS- } \\
\text { PECTS } \geq 6\end{array}$ \\
\hline $\begin{array}{l}\text { Onset to randomization/groin } \\
\text { puncture/first reperfusion (minute) }\end{array}$ & 146/NA/225 & 330/381/NA & $\quad 146 / 2$ & $8 / 244$ & 200/260/NA & 170/185/241 & 169/210/NA & 185/224/252 & 225/269/355 \\
\hline ERT arm & IA only & $\begin{array}{l}\text { IA with } \\
\text { standard care }\end{array}$ & IA with & IV-TPA & $\begin{array}{l}\text { IA with } \\
\text { standard care }\end{array}$ & $\begin{array}{l}\text { A with standard } \\
\text { care }\end{array}$ & IA with IV-TPA & IA with IV-TPA & $\begin{array}{l}\text { IA with } \\
\text { standard care }\end{array}$ \\
\hline Control arm & IV-TPA & Standard care & & PA & Standard care & Standard care & IV-TPA & IV-TPA & Standard care \\
\hline IV-TPA (active/control) (\%) & 0/98.3 & $43.8 / 29.6$ & 100 & 100 & 87.1/90.6 & 72.7/78.7 & $100 / 100$ & $100 / 100$ & 68.0/77.0 \\
\hline ERT performed in ERT arm (\%) & 91.2 & 95.3 & & 0 & 83.7 & 91.5 & 85.7 & 88.8 & 95.1 \\
\hline $\begin{array}{l}\text { Stent-Retriever thrombectomy } \\
\text { in active arm (\%) }\end{array}$ & 12.7 & 0.0 & & & 81.5 & 78.8 & 77.1 & 88.8 & 95.1 \\
\hline mTICI 2b-3 in active arm (\%) & NA & 25.0 & & & 58.7 & 72.4 & 86.2 & 88.0 & 65.7 \\
\hline Outcome assessment (day) & 90 & 90 & & 0 & 90 & 90 & 90 & 90 & 90 \\
\hline
\end{tabular}

*Imaging entry criteria were changed from original criteria (Infarct core $\leq 50 \mathrm{~mL}$, severe hypoperfusion $\leq 100 \mathrm{~mL}$, ischemic penumbra $<15 \mathrm{~mL}$ and mismatch ratio $\leq 1.8$ ). ERT, endovascular recanalization therapy; NA, not assessed; LAO, large artery occlusion; ICA, internal carotid artery; BA, basilar artery; VA, vertebral artery; UK, urokinase; IV, intravenous; mTICl, modified Thrombolysis in Cerebral Infarction; mRS, modified Rankin Scale; OR, odds ratio; Cl, confidence interval; SICH, symptomatic intracranial hemorrhage; IA, intra-arterial; TPA, tissue type plasminogen activator; MCA, middle cerebral artery; ASPECTS, Alberta Stroke Program Early CT Score; NA, not available. 
Risk of bias graph for all in cluded trials

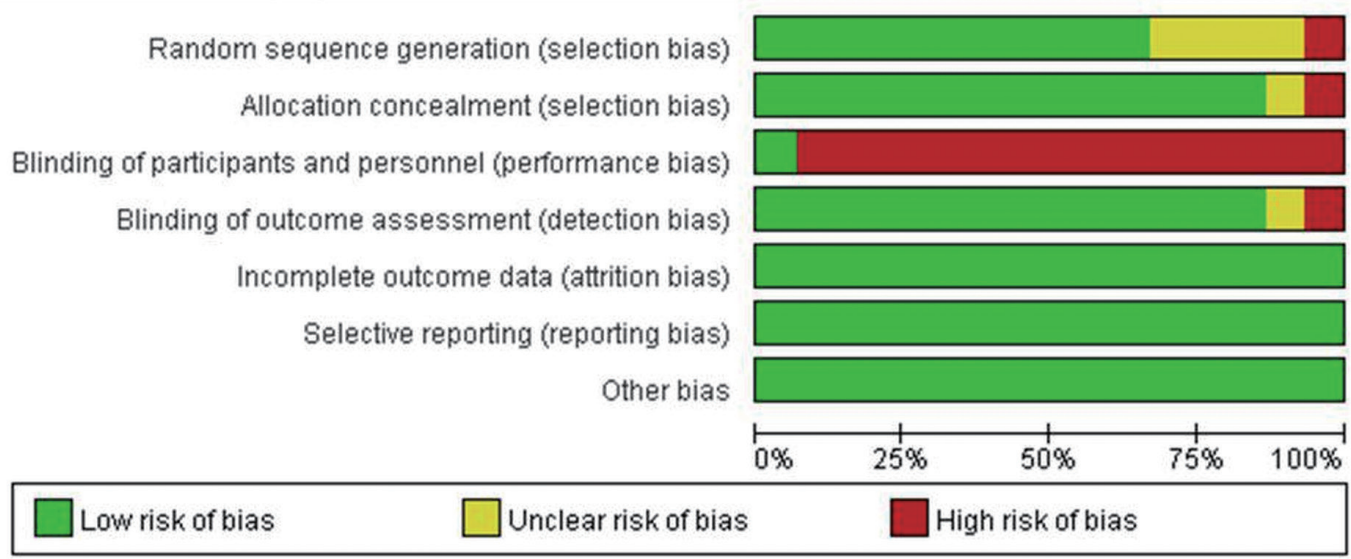

Risk of bias summary for each trial

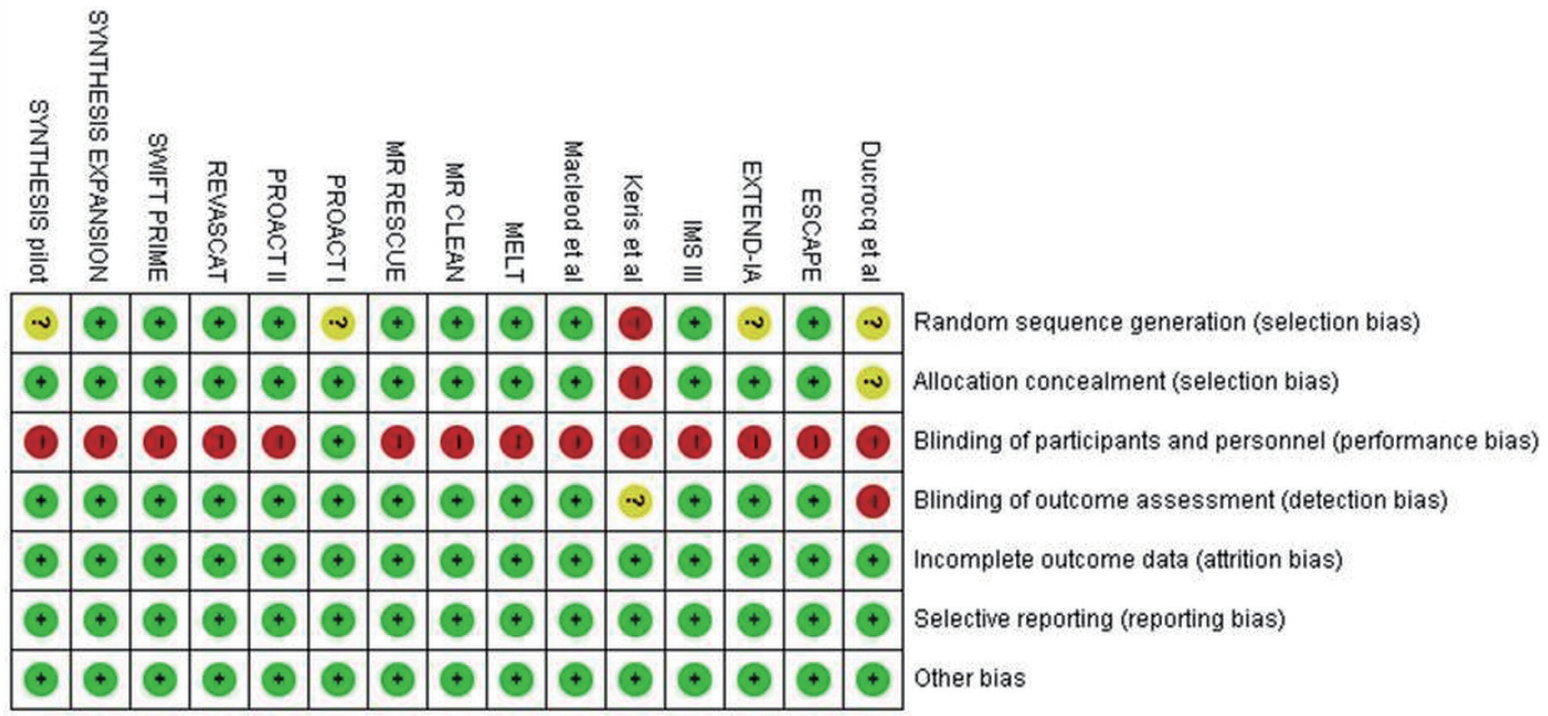

B

Figure 2. Bias risk assessment for each trial.

Table 2. Effect of IA thrombolysis on end points from all 15 trials

\begin{tabular}{|c|c|c|c|c|c|c|c|}
\hline End points & No. of trials & Active, n/N (\%) & Control, n/N (\%) & Pooled OR (95\% CI) & $P$ value & $\begin{array}{l}\text { Test for publication bias } \\
\text { (Begg's test) }\end{array}$ & NNT or NNH \\
\hline mRS 0-2 & 13 & $657 / 1,517(43.3)$ & 402/1,261 (31.9) & $1.79(1.34,2.40)$ & $<0.001$ & 0.8072 & 9 \\
\hline mRS 0-1 & 13 & $435 / 1,530(28.4)$ & $245 / 1,261(19.4)$ & $1.81(1.34,2.44)$ & $<0.001$ & 0.7143 & 11 \\
\hline mRS 0-3 & 13 & $907 / 1,516$ (59.8) & $617 / 1,280(48.2)$ & $1.66(1.25,2.22)$ & 0.001 & 0.6255 & 9 \\
\hline Good NIHSS outcome & 5 & 156/392 (39.7) & $68 / 299(22.7)$ & $3.11(2.14,4.53)$ & $<0.001$ & 0.6242 & 6 \\
\hline Good Bl outcome & 7 & $334 / 677(49.3)$ & 190/622 (30.5) & $2.24(1.78,2.82)$ & $<0.001$ & 0.8806 & 5 \\
\hline mAOL, TIMI, or TICI 2-3 & 6 & 429/568 (75.6) & $227 / 489(46.4)$ & $4.50(1.97,10.27)$ & $<0.001$ & 0.5730 & 3 \\
\hline mAOL, TIMI, or TICl 3 & 3 & 167/321 (51.9) & 71/271 (26.2) & $6.21(4.05,9.50)$ & $<0.001$ & 0.6015 & 4 \\
\hline Any ICH & 10 & $341 / 1,053(32.4)$ & $150 / 770(19.5)$ & $1.93(1.37,2.72)$ & $<0.001$ & 0.9287 & 8 \\
\hline Symptomatic ICH & 15 & $90 / 1,562$ (5.8) & $61 / 1,318(4.6)$ & $1.19(0.83,1.69)$ & 0.345 & 0.8695 & 88 \\
\hline mRS 5-6 & 12 & $366 / 1,504$ (24.3) & $365 / 1,251(29.2)$ & $0.77(0.61,0.97)$ & 0.025 & 0.7839 & 21 \\
\hline Mortality & 15 & $273 / 1,555(17.6)$ & $254 / 1,312(19.4)$ & $0.87(0.71,1.05)$ & 0.151 & 0.6560 & 55 \\
\hline
\end{tabular}

n/N: number of patients achieving each end point/number of patients included. OR, odds ratio; NNT, number needed to treat; NNH, number needed to harm; mRS, modified Rankin Scale; NIHSS, National Institute of Health Stroke Scale; mAOL, modified arterial occlusive lesion; TIMI, Thrombolysis in Myocardial Infarction; TICI, Thrombolysis in Cerebral Infarction; ICH, intracerebral hemorrhage. 
Table 3. Effect of IA thrombolysis on end points in 5 stent-retriever trials

\begin{tabular}{|c|c|c|c|c|c|c|c|}
\hline End points & No. of trials & Active, n/N (\%) & Control, n/N (\%) & Pooled OR (95\% CI) & $P$ value & $\begin{array}{l}\text { Test for publication bias } \\
\text { (Begg's test) }\end{array}$ & NNT or NNH \\
\hline mRS 0-2 & 5 & 292/633 (46.1) & $170 / 645(26.4)$ & $2.39(1.88,3.04)$ & $<0.001$ & 0.1416 & 5 \\
\hline mRS 0-1 & 5 & 170/633 (26.9) & $83 / 645(12.9)$ & $2.49(1.85,3.36)$ & $<0.001$ & 0.6242 & 7 \\
\hline mRS 0-3 & 5 & 399/633 (63.0) & $276 / 645(42.8)$ & $2.33(1.72,3.15)$ & $<0.001$ & 0.1416 & 5 \\
\hline Favorable shift on mRS score & 5 & - & - & $2.02(1.64,2.48)$ & $<0.001$ & 0.6242 & - \\
\hline Good NIHSS outcome & 2 & 109/188 (58.0) & $52 / 169(30.8)$ & $3.62(2.26,5.78)$ & $<0.001$ & Not measurable & 4 \\
\hline Good Bl outcome & 3 & $240 / 460(52.2)$ & 145/478 (30.3) & $2.53(1.83,3.52)$ & $<0.001$ & 0.1172 & 5 \\
\hline mAOL, TIMI, or TICl 2-3 & 3 & $303 / 378$ (80.2) & $177 / 380(46.6)$ & $5.68(3.09,10.45)$ & $<0.001$ & 0.1172 & 3 \\
\hline mAOL, TIMl, or TICl 3 & 1 & $141 / 187(75.4)$ & 70/207 (33.8) & $6.00(3.86,9.32)$ & $<0.001$ & Not measurable & 2 \\
\hline Any ICH & 4 & 98/401 (24.4) & $57 / 385(14.8)$ & $1.48(0.76,2.91)$ & 0.252 & 0.1742 & 10 \\
\hline Symptomatic ICH & 5 & 26/634 (4.1) & 28/652 (4.3) & $1.08(0.61,1.88)$ & 0.798 & 0.0500 & 517 \\
\hline mRS 5-6 & 5 & 137/633 (21.6) & 209/649 (32.2) & $0.57(0.41,0.78)$ & 0.001 & 0.1416 & 9 \\
\hline Mortality & 5 & 97/633 (15.3) & $122 / 649$ (18.8) & $0.78(0.54,1.12)$ & 0.177 & 0.6242 & 29 \\
\hline
\end{tabular}

n/N: number of patients achieving each end point/number of patients included. OR, odds ratio; NNT, number needed to treat; NNH, number needed to harm; mRS, modified Rankin Scale; NIHSS, National Institute of Health Stroke Scale; BI, Barthel index; mAOL, modified arterial occlusive lesion; TIMI, Thrombolysis in Myocardial Infarction; TICI, Thrombolysis in Cerebral Infarction; ICH, intracerebral hemorrhage.

Table 4. Effect of IA thrombolysis versus IVTPA on end points in 8 trials of IV-TPA rate $>75 \%$ in the control arms

\begin{tabular}{|c|c|c|c|c|c|c|c|}
\hline End points & No. of trials & Active, n/N (\%) & Control, n/N (\%) & Pooled OR (95\% CI) & $P$-value & $\begin{array}{l}\text { Test for publication bias } \\
\text { (Begg's test) }\end{array}$ & NNT or NNH \\
\hline mRS 0-2 & 8 & $559 / 1,254(44.6)$ & $349 / 1,069(32.6)$ & $1.88(1.29,2.73)$ & 0.001 & 0.4579 & 8 \\
\hline mRS 0-1 & 8 & $359 / 1,254$ (28.6) & 212/1,069 (19.8) & $1.82(1.24,2.69)$ & 0.002 & 0.4579 & 11 \\
\hline mRS 0-3 & 8 & 776/1,254 (61.9) & $524 / 1,069(49.0)$ & $1.83(1.31,2.56)$ & 0.001 & 0.2160 & 8 \\
\hline Good NIHSS outcome & 2 & 109/188 (58.0) & $52 / 169$ (30.8) & $3.62(2.26,5.78)$ & $<0.001$ & Not measurable & 4 \\
\hline Good Bl outcome & 3 & $240 / 460(52.2)$ & 145/478 (30.3) & $2.53(1.83,3.52)$ & $<0.001$ & 0.1172 & 5 \\
\hline mAOL, TIMI, or TICI 2-3 & 3 & $303 / 378(80.2)$ & 177/380 (46.6) & $5.68(3.09,10.45)$ & $<0.001$ & 0.1172 & 3 \\
\hline mAOL, TIMI, or TICI 3 & 1 & 141/187 (75.4) & 70/207 (33.8) & $6.00(3.86,9.32)$ & $<0.001$ & Not measurable & 2 \\
\hline Any ICH & 5 & 244/835 (29.2) & 112/607 (18.5) & $1.57(1.02,2.43)$ & 0.042 & 0.3272 & 9 \\
\hline Symptomatic ICH & 8 & 65/1,274 (5.1) & $55 / 1,084(5.1)$ & $1.03(0.70,1.50)$ & 0.893 & 0.0478 & 3,541 \\
\hline mRS 5-6 & 8 & $282 / 1,254$ (22.5) & $308 / 1,073(28.7)$ & $0.70(0.52,0.93)$ & 0.013 & 0.4579 & 16 \\
\hline Mortality & 8 & $212 / 1,254$ (16.9) & 193/1,073 (18.0) & $0.90(0.69,1.17)$ & 0.432 & 0.6207 & 93 \\
\hline
\end{tabular}

n/N: number of patients achieving each end point/number of patients included. OR, odds ratio; NNT, number needed to treat; NNH, number needed to harm; mRS, modified Rankin Scale; NIHSS, National Institute of Health Stroke Scale; mAOL, modified arterial occlusive lesion; TIMI, Thrombolysis in Myocardial Infarction; TICI, Thrombolysis in Cerebral Infarction; ICH, intracerebral hemorrhage.

\section{Discussion}

This updated meta-analysis, including all relevant published trials to date, clearly demonstrates the benefits of ERT in patients with AIS caused by large artery occlusion. ERT significantly increased good outcome (mRS 0-2) and excellent outcome (mRS 0-1) in parallel with increasing good neurological and activity of daily living outcomes. For all trials including patients with no IV-TPA and IV-TPA in the control arms, ERT increased these outcomes by1.8- to 3.1-fold. When separately analyzing trials in which the majority of cases were treated with IV-TPA in the control arms, the magnitudes of ERT's benefits were similar. In particular, the benefits were pronounced in recent stent-retriever trials, showing that the chances of achieving these outcomes more than doubled or tripled with ERT. In addition, patients treated with stent-retriever thrombectomy had about a 2-fold odds of a favorable shift in the mRS disability score. Beyond statistical significance, the treatment effects were substantial. The NNTs for achieving one additional outcome of mRS 0-2 and mRS 0-1 were 9 and 11 for all trials. The NNT values were even lower with stent-retriever trials, in which $88 \%$ of patients assigned to control arms received IV-TPA therapy: for every five patients who were treated with stent-retriever in addition to IV-TPA therapy, one additional patient achieved an mRS 0-2 outcome; for every seven patients, one additional patient had no or minimal disability (mRS 0-1).

ERT substantially increased reperfusion rates, which likely accounted for the clinical benefits observed. The absolute increase in partial or complete recanalization rate with ERT compared to control was $29.1 \%$. In stent-retriever trials, the rate of partial or complete recanalization with ERT was $80.2 \%$, and the absolute increase compared to IV-TPA ( $88 \%$ treated with IV- 
Pooling all RCTs

\begin{tabular}{|c|c|c|c|c|c|c|c|c|c|c|c|c|}
\hline Stucty or Subqroup & \multicolumn{2}{|c|}{ ERT } & \multicolumn{2}{|c|}{ Control } & \multicolumn{3}{|c|}{ Odds Ratio } & \multicolumn{5}{|c|}{$\begin{array}{c}\text { Odds Ratio } \\
\text { M.H. Random. } 95 \% \mathrm{Cl}\end{array}$} \\
\hline PROACT॥ & 48 & 121 & 15 & 59 & $8.1 \%$ & $1.93[0.97,3.85]$ & 1999 & & & & & \\
\hline Ducrocq et al & 6 & 13 & 4 & 14 & $2.7 \%$ & $2.14[0.44,10.53]$ & 2005 & & & & & \\
\hline Macleod et al & 4 & 8 & 1 & 8 & $1.2 \%$ & $7.00[0.57,86.32]$ & 2005 & & & & & \\
\hline MELT & 28 & 57 & 22 & 57 & $7.5 \%$ & $1.54[0.73,3.23]$ & 2007 & & & & & \\
\hline SYNTHESIS pilot & 14 & 25 & 9 & 29 & $4.7 \%$ & $2.83[0.93,8.62]$ & 2009 & & & & & \\
\hline SYNTHESIS EXPANSION & 76 & 181 & 84 & 181 & $11.3 \%$ & $0.84[0.55,1.27]$ & 2013 & & & & & \\
\hline MR RESCUE & 12 & 64 & 11 & 54 & $6.0 \%$ & $0.90[0.36,2.25]$ & 2013 & & & & & \\
\hline IMS III & 177 & 415 & 86 & 214 & $12.3 \%$ & $1.11[0.79,1.55]$ & 2013 & & & & & \\
\hline MR CLEAN & 76 & 233 & 51 & 267 & $11.4 \%$ & $2.05[1.36,3.09]$ & 2014 & & & & & \\
\hline REVASCAT & 45 & 103 & 29 & 103 & $9.3 \%$ & $1.98[1.11,3.53]$ & 2015 & & & & & \\
\hline SWIFT PRIME & 59 & 98 & 33 & 93 & $9.2 \%$ & $2.75[1.53,4.94]$ & 2015 & & & & & \\
\hline ESCAPE & 87 & 164 & 43 & 147 & $10.7 \%$ & $2.73[1.71,4.37]$ & 2015 & & & & & \\
\hline EXTEND-IA & 25 & 35 & 14 & 35 & $5.4 \%$ & $3.75[1.38,10.17]$ & 2015 & & & & & \\
\hline Total $(95 \% \mathrm{Cl})$ & & 1517 & & 1261 & $100.0 \%$ & $1.79[1.34,2.40]$ & & & & & & \\
\hline Total events & 657 & & 402 & & & & & & & & & \\
\hline $\begin{array}{l}\text { Heterogeneity: } \operatorname{Tau}^{2}=0.15 \\
\text { Test for overall effect: } Z=3\end{array}$ & $\begin{array}{l}\mathrm{Chi}^{2}=31 \\
1(\mathrm{P}<0 .\end{array}$ & $\begin{array}{l}28, \mathrm{df}= \\
0001)\end{array}$ & $=12(P=$ & $0.002)$ & $F^{2}=62 \%$ & & & $\begin{array}{ll}0.1 & 0.2 \\
& F\end{array}$ & urs Control & Favours ERT & 5 & $10^{\prime}$ \\
\hline
\end{tabular}

Pooling stent-retriever RCTs

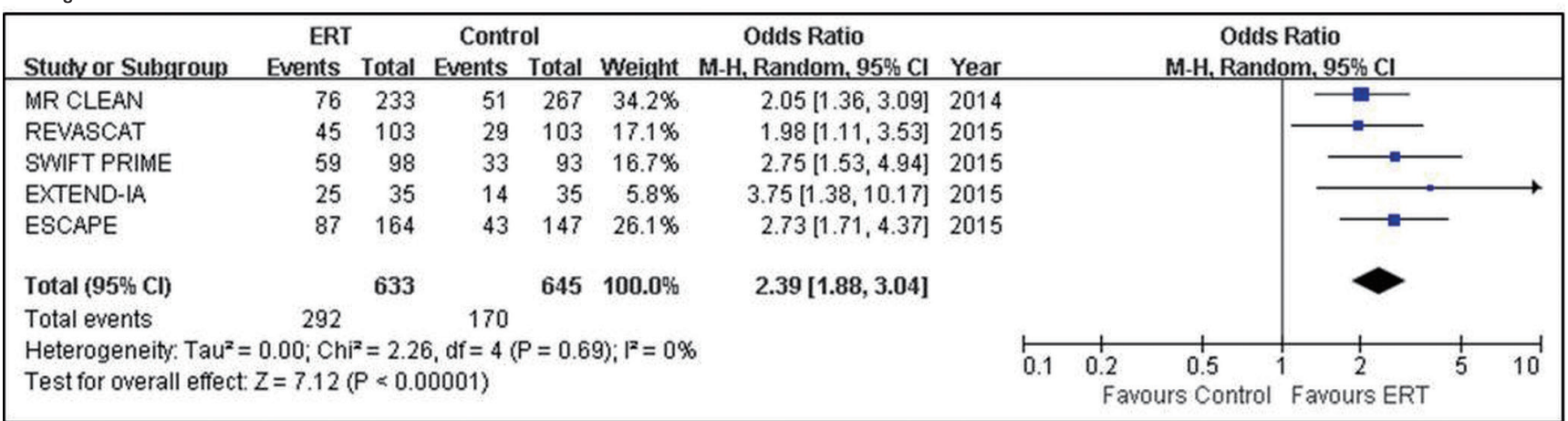

Pooling RCTs with IV-TPA rate $>75 \%$ in control arms

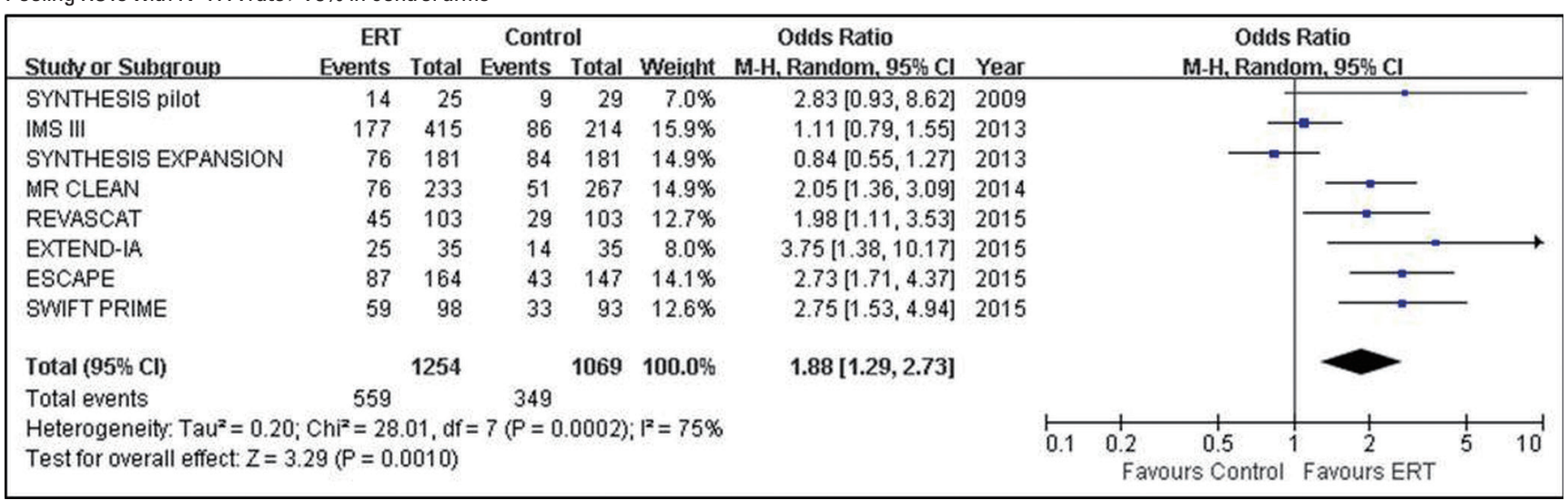

Figure 3. Pooled estimates for achieving modified Rankin Scale (mRS) 0-2 outcomes with Endovascular recanalization therapy vs. control.

TPA) was 33.6\%. Therefore, for every three patients, stent-retriever ERT in addition to IV-TPA would achieve one additional recanalization.

Although ERT was associated with an increased risk of any ICH, ERT did not increase SICH and mortality in all trials, stent-retriever trials, and RCTs including a control arm with IV-
TPA. Therefore, most ICHs were radiological and asymptomatic. In contrast, ERT significantly decreased the rates of extreme disability or death (mRS 5-6) in all analyses. For stroke outcomes, combining extreme disability and mortality seems to be more clinically meaningful than mortality alone. Laypersons as well as stroke experts perceived mRS 5 disability as bad as or 
Pooling all RCTs

\begin{tabular}{|c|c|c|c|c|c|c|c|c|c|c|c|c|}
\hline Stucty or Subgroup & $\begin{array}{l}\text { ERT } \\
\text { Events }\end{array}$ & Total & $\begin{array}{l}\text { Contr } \\
\text { Events }\end{array}$ & $\begin{array}{l}\text { ol } \\
\text { Total }\end{array}$ & Weight & $\begin{array}{l}\text { Odds Ratio } \\
\text { M-H. Random. } 95 \% \text { Cl }\end{array}$ & Year & & $\begin{array}{r}\text { Odds } \\
\text { M-H, Rando }\end{array}$ & $\begin{array}{l}\text { Ratio } \\
\text { om. } 95 \% \mathrm{Cl}\end{array}$ & & \\
\hline PROACTI & 8 & 26 & 3 & 14 & $3.2 \%$ & $1.63[0.35,7.48]$ & 1998 & & & & & \\
\hline PROACTIII & 32 & 121 & 10 & 59 & $7.8 \%$ & $1.76[0.80,3.89]$ & 1999 & & & & & \\
\hline Macleod et al & 3 & 8 & 0 & 8 & $0.9 \%$ & $10.82[0.46,252.79]$ & 2005 & & & & & \\
\hline MELT & 24 & 57 & 13 & 57 & $7.6 \%$ & $2.46[1.09,5.54]$ & 2007 & & & & & \\
\hline SYNTHESIS pilot & 12 & 25 & 8 & 29 & $5.0 \%$ & $2.42[0.78,7.51]$ & 2009 & & & & & \\
\hline IMS III & 122 & 415 & 58 & 214 & $13.5 \%$ & $1.12[0.78,1.62]$ & 2013 & & & & & \\
\hline MR RESCUE & 9 & 64 & 7 & 54 & $5.4 \%$ & $1.10[0.38,3.18]$ & 2013 & & & & & \\
\hline SYNTHESIS EXPANSION & 55 & 181 & 63 & 181 & $12.4 \%$ & $0.82[0.53,1.27]$ & 2013 & & & & & \\
\hline MR CLEAN & 27 & 233 & 16 & 267 & $9.5 \%$ & $2.06[1.08,3.92]$ & 2014 & & & & & \\
\hline ESCAPE & 58 & 164 & 26 & 147 & $11.1 \%$ & $2.55[1.50,4.33]$ & 2015 & & & & & \\
\hline EXTEND-IA & 18 & 35 & 10 & 35 & $6.0 \%$ & $2.65[0.99,7.11]$ & 2015 & & & & & \\
\hline REVASCAT & 25 & 103 & 13 & 103 & $8.4 \%$ & $2.22[1.06,4.63]$ & 2015 & & & & & \\
\hline SWIFT PRIME & 42 & 98 & 18 & 93 & $9.4 \%$ & $3.13[1.63,6.00]$ & 2015 & & & & & \\
\hline Total $(95 \% \mathrm{Cl})$ & & 1530 & & 1261 & $100.0 \%$ & $1.81[1.34,2.44]$ & & & & & & \\
\hline Total events & 435 & & 245 & & & & & & & & & \\
\hline $\begin{array}{l}\text { Heterogeneity: } \operatorname{Tau}^{2}=0.14 \\
\text { Test for overall effect: } Z=3\end{array}$ & $\begin{array}{l}C h i^{2}=25 \\
6(P=0 .\end{array}$ & $\begin{array}{l}43, d f= \\
0001)\end{array}$ & $12(P=$ & $0.01) ; 1$ & $z=53 \%$ & & & $\begin{array}{ll}0.1 & 0.2\end{array}$ & 0.51 & $1 \frac{2}{2}$ & 5 & 10 \\
\hline
\end{tabular}

Pooling stent-retriever RCTs

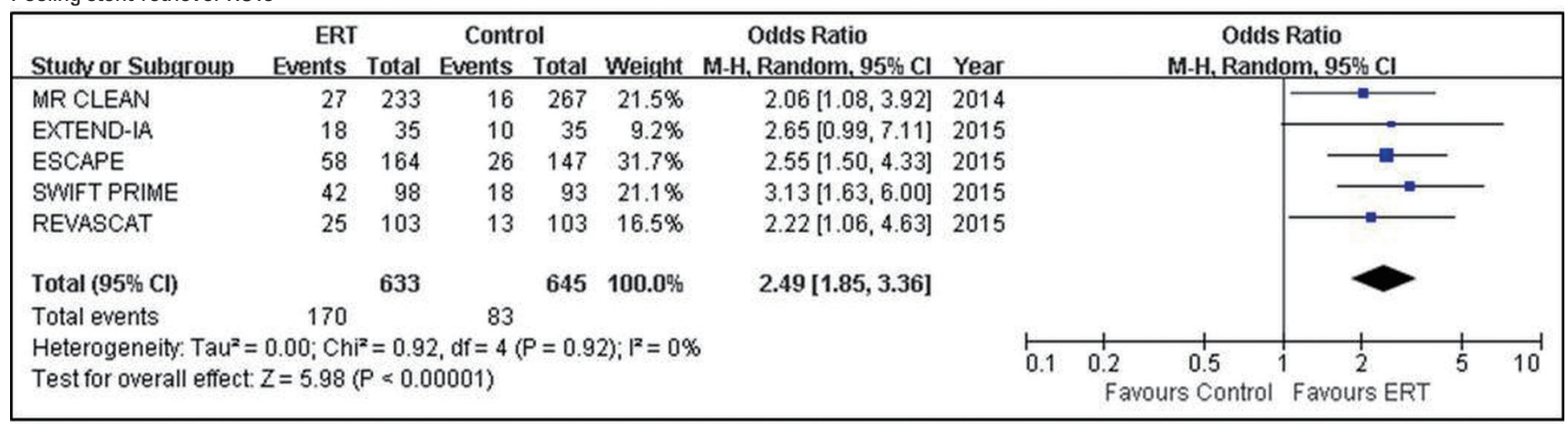

Pooling RCTs with IV-TPA rate $>75 \%$ in control arms

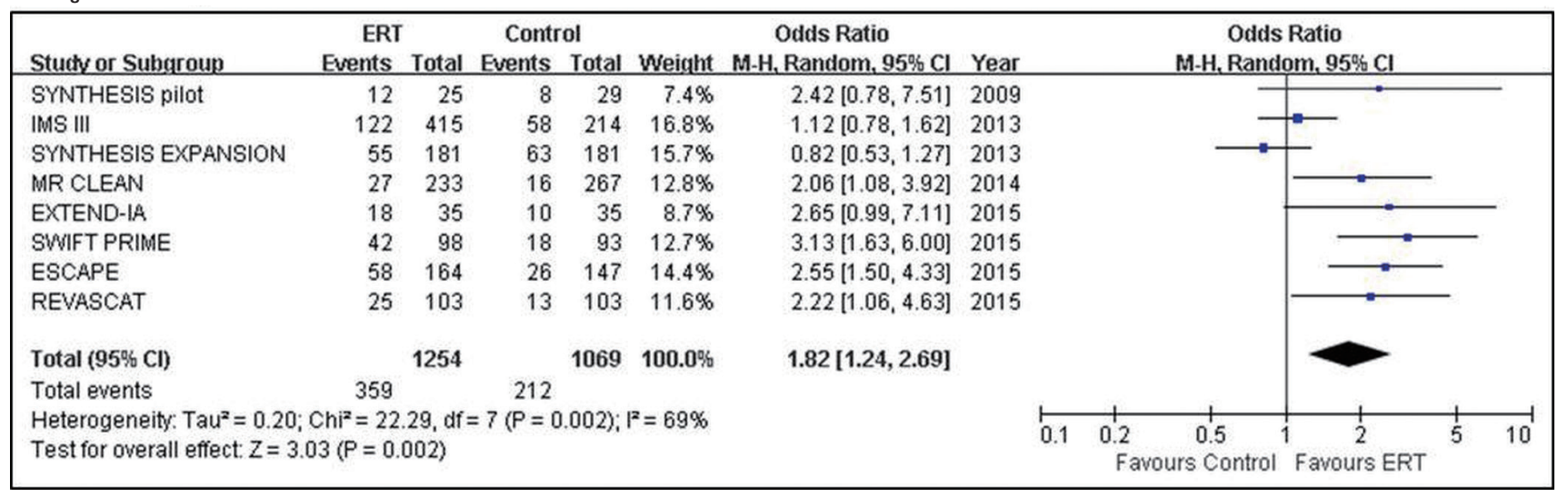

Figure 4. Pooled estimates for achieving modified Rankin Scale (mRS) 0-1 outcome with Endovascular recanalization therapy vs. control.

even worse than death, ${ }^{21,22}$ and therefore extreme disability and death are frequently collapsed into a single worst-outcome category in acute stroke trials. ${ }^{10,23}$

It would be instructive to compare the magnitude of the ERT's benefits with those of coronary interventions. In a metaanalysis of RCTs comparing primary percutaneous coronary in- tervention with intravenous fibrinolytic therapy in ST-segmentelevation myocardial infarction, the NNT was 45 to prevent one short-term mortality and 29 to prevent long-term mortality. ${ }^{24}$ When pooling five recent stent-retriever trials, the NNT to avoid one mortality at 3 months with ERT compared to standard therapy (IV-TPA in most cases) was 29, although the reduction was 
Pooling all RCTs

\begin{tabular}{|c|c|c|c|c|c|c|c|c|c|c|c|}
\hline Stucty or Subqroup & $\begin{array}{l}\text { ERT } \\
\text { Events }\end{array}$ & Total & $\begin{array}{l}\text { Contr } \\
\text { Events }\end{array}$ & Tot & Weight & $\begin{array}{l}\text { Odds Ratio } \\
\text { M-H. Random, } 95 \% \mathrm{Cl}\end{array}$ & Year & & $\begin{aligned} \text { Odds } \\
\text { M-H, Rando }\end{aligned}$ & $\begin{array}{l}\text { Ratio } \\
\text { om. } 95 \% \mathrm{Cl}\end{array}$ & \\
\hline PROACTI & 15 & 26 & 2 & 14 & $11.5 \%$ & $8.18[1.51,44.21]$ & 1998 & & & & $\longrightarrow$ \\
\hline PROACT ॥ & 71 & 108 & 9 & 50 & $18.3 \%$ & $8.74[3.84,19.92]$ & 1999 & & & & \\
\hline MR RESCUE & 40 & 56 & 39 & 45 & $16.5 \%$ & $0.38[0.14,1.08]$ & 2013 & & & & \\
\hline MR CLEAN & 157 & 187 & 119 & 207 & $20.8 \%$ & $3.87[2.40,6.24]$ & 2014 & & & & \\
\hline EXTEND-IA & 33 & 35 & 15 & 35 & $12.3 \%$ & $22.00[4.55,106.43]$ & 2015 & & & & $\rightarrow$ \\
\hline ESCAPE & 113 & 156 & 43 & 138 & $20.6 \%$ & $5.81[3.51,9.60]$ & 2015 & & & & \\
\hline Total $(95 \% \mathrm{Cl})$ & & 568 & & 489 & $100.0 \%$ & $4.50[1.97,10.27]$ & & & & & \\
\hline Total events & 429 & & 227 & & & & & & & & \\
\hline \multicolumn{8}{|c|}{$\begin{array}{l}\text { Heterogeneity: } \text { Tau }^{2}=0.79 ; \mathrm{Chi}^{2}=29.90, \mathrm{df}=5(\mathrm{P}<0.0001) ; \mathrm{I}^{2}=83 \% \\
\text { Test for overall effect: } \mathrm{Z}=3.58(\mathrm{P}=0.0003)\end{array}$} & 0.05 & 0.21 & & 20 \\
\hline
\end{tabular}

Pooling stent-retriever RCTs

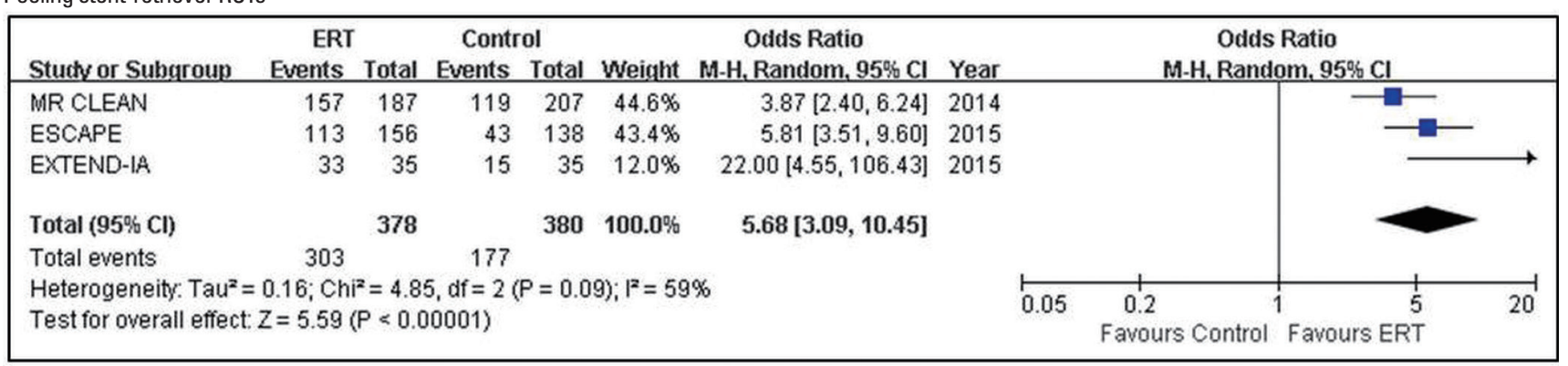

Pooling RCTs with IV-TPA rate $>75 \%$ in control arms

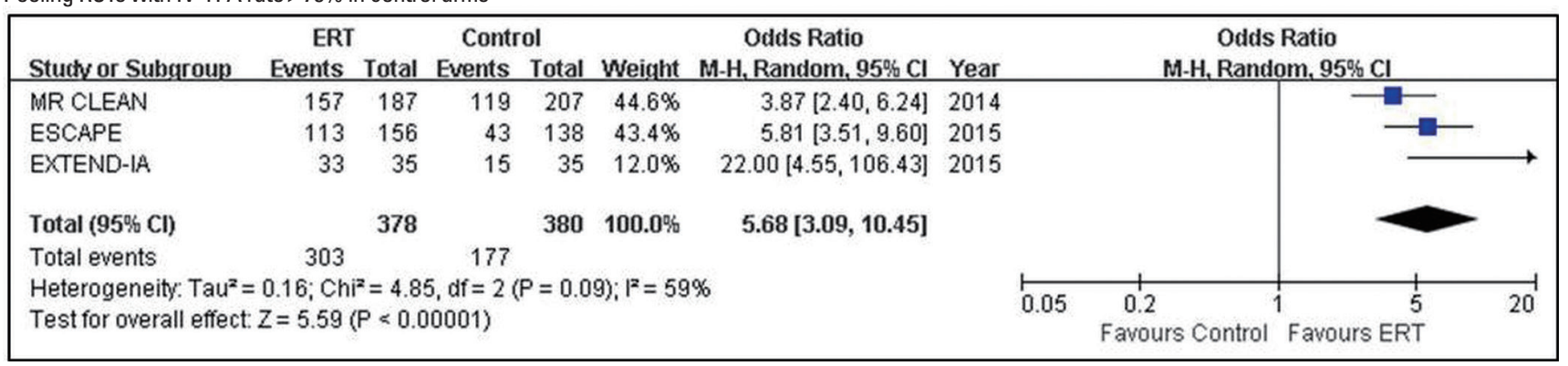

Figure 5. Pooled estimates for achieving partial or complete recanalization with Endovascular recanalization therapy vs. control.

not statistically significant. Moreover, the NNT to prevent one patient from having an mRS 5-6 outcome was only 9, and this reduction was highly statistically significant. Therefore, the benefits of ERT with stent-retriever in severe AIS appear to be greater than the benefits of primary percutaneous coronary intervention in ST-segment-elevation myocardial infarction.

Our findings accord with and extend the results of an earlier meta-analysis. ${ }^{5}$ The earlier meta-analysis compared ERT with no thrombolytic therapy using data from five RCTs involving 395 patients. Our study included 15 RCTs involving 2,899 patients, and also compared ERT with contemporary standard therapy largely composed of IV-TPA. Therefore, our results more convincingly indicate the benefits of ERT in the current clinical setting.

For most efficacy outcomes, heterogeneity was present among trials. However, this heterogeneity was mainly driven by variations in the magnitude rather than in the direction of the treatment effect. For SICH, there was no heterogeneity for extreme disability or death and mortality outcomes. In addition, when restricting analysis to stent-retriever trials, we found no significant heterogeneity across the trials for most outcomes except for any $\mathrm{ICH}$ and partial or complete recanalization rates. Therefore, the benefit of ERT appears generally consistent across trials, particularly among the stent-retriever trials.

The validity of meta-analysis results can only be guaranteed when the quality of included trials is high. Most RCTs included in this meta-analysis were at low risk of bias for each item except for treatment blinding. However, blinding of treatment assignments in trials comparing endovascular thrombectomy versus medical therapy would not be feasible for practical reason. Most 
Pooling all RCTs

\begin{tabular}{|c|c|c|c|c|c|c|c|c|c|c|c|c|}
\hline Stucty or Subgroup & $\begin{array}{l}\text { ERT } \\
\text { Events }\end{array}$ & Total & $\begin{array}{l}\text { Contro } \\
\text { Events }\end{array}$ & $\begin{array}{l}\text { ol } \\
\text { Total }\end{array}$ & Weight & $\begin{array}{l}\text { Odds Ratio } \\
\text { M-H, Random, } 95 \% \mathrm{Cl}\end{array}$ & Year & & $\begin{array}{r}\text { Odds F } \\
\text { M-H, Rando }\end{array}$ & $\begin{array}{l}\text { Ratio } \\
\text { am, } 95 \% \mathrm{Cl}\end{array}$ & & \\
\hline PROACTI & 4 & 26 & 1 & 14 & $2.4 \%$ & $2.36[0.24,23.48]$ & 1998 & & & & & \\
\hline PROACT II & 11 & 108 & 1 & 54 & $2.9 \%$ & $6.01[0.76,47.84]$ & 1999 & & & & & \\
\hline Keris et al & 0 & 12 & 1 & 33 & $1.2 \%$ & $0.87[0.03,22.72]$ & 2001 & & & & & \\
\hline Ducrocq et al & 2 & 13 & 0 & 14 & $1.3 \%$ & $6.30[0.27,144.70]$ & 2005 & & & & & \\
\hline Macleod et al & 0 & 8 & 0 & 8 & & Not estimable & 2005 & & & & & \\
\hline MELT & 5 & 57 & 1 & 57 & $2.6 \%$ & $5.38[0.61,47.63]$ & 2007 & & & & & \\
\hline SYNTHESIS pilot & 2 & 25 & 4 & 29 & $3.9 \%$ & $0.54[0.09,3.25]$ & 2009 & & & & & \\
\hline MRRESCUE & 3 & 64 & 2 & 54 & $3.7 \%$ & $1.28[0.21,7.95]$ & 2013 & & & & & \\
\hline SYNTHESIS EXPANSION & 10 & 181 & 10 & 181 & $15.3 \%$ & $1.00[0.41,2.46]$ & 2013 & & & & & \\
\hline IMS III & 27 & 434 & 13 & 222 & $26.8 \%$ & $1.07[0.54,2.11]$ & 2013 & & & & & \\
\hline MR CLEAN & 18 & 233 & 17 & 267 & $26.4 \%$ & $1.23[0.62,2.45]$ & 2014 & & & & & \\
\hline EXTEND-IA & 0 & 35 & 2 & 35 & $1.3 \%$ & $0.19[0.01,4.08]$ & 2015 & $\leftarrow$ & & & & \\
\hline SWIFT PRIME & 0 & 98 & 3 & 97 & $1.4 \%$ & $0.14[0.01,2.69]$ & 2015 & $\leftarrow$ & & & & \\
\hline ESCAPE & 6 & 165 & 4 & 150 & $7.6 \%$ & $1.38[0.38,4.98]$ & 2015 & & & & & \\
\hline REVASCAT & 2 & 103 & 2 & 103 & $3.2 \%$ & $1.00[0.14,7.24]$ & 2015 & & & & & \\
\hline Total $(95 \% \mathrm{Cl})$ & & 1562 & & 1318 & $100.0 \%$ & $1.19[0.83,1.69]$ & & & & & & \\
\hline Total events & 90 & & 61 & & & & & & & & & \\
\hline \multicolumn{8}{|c|}{$\begin{array}{l}\text { Heterogeneity: } \text { Tau }^{2}=0.00 ; C h i^{2}=10.15, d f=13(P=0.68) ; I^{2}=0 \% \\
\text { Test for overall effect: } Z=0.94(P=0.35)\end{array}$} & $0.1 \quad 0.2$ & $\begin{array}{c}0.5 \\
\text { Favours ERT }\end{array}$ & $\begin{array}{cc}2 & 5 \\
\text { Favours Control }\end{array}$ & 5 & 10 \\
\hline
\end{tabular}

Pooling stent-retriever RCTs

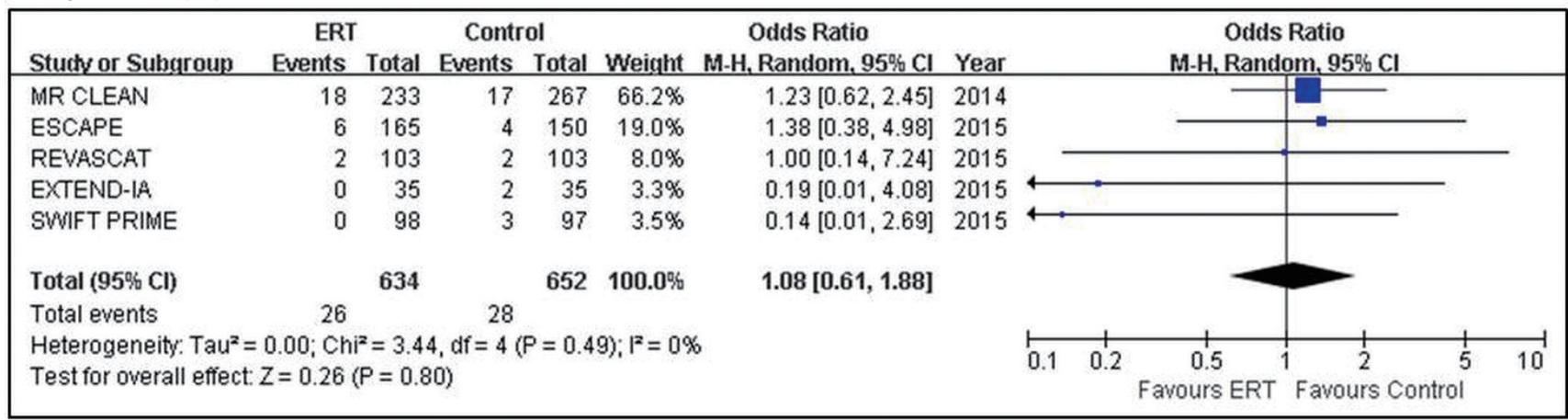

Pooling RCTs with IV-TPA rate $>75 \%$ in control arms

\begin{tabular}{|c|c|c|c|c|c|c|c|c|c|c|c|c|}
\hline Stucty or Subqroup & $\begin{array}{l}\text { ERT } \\
\text { Events }\end{array}$ & Iotal & $\begin{array}{l}\text { Contr } \\
\text { Events }\end{array}$ & $\begin{array}{l}\text { rol } \\
\text { Total }\end{array}$ & Weight & $\begin{array}{c}\text { Odds Ratio } \\
\text { M-H, Random, } 95 \% \mathrm{Cl}\end{array}$ & Year & & $\begin{array}{r}\text { Odds F } \\
\text { M-H, Rando }\end{array}$ & $\begin{array}{l}\text { Ratio } \\
\text { om, } 95 \% \mathrm{Cl}\end{array}$ & & \\
\hline SYNTHESIS pilot & 2 & 25 & 4 & 29 & $4.5 \%$ & $0.54[0.09,3.25]$ & 2009 & 4 & & & & \\
\hline SYNTHESIS EXPANSION & 10 & 181 & 10 & 181 & $17.9 \%$ & $1.00[0.41,2.46]$ & 2013 & & & & & \\
\hline |MS ||| & 27 & 434 & 13 & 222 & $31.2 \%$ & $1.07[0.54,2.11]$ & 2013 & & & & & \\
\hline MR CLEAN & 18 & 233 & 17 & 267 & $30.7 \%$ & $1.23[0.62,2.45]$ & 2014 & & & & & \\
\hline SWIFT PRIME & 0 & 98 & 3 & 97 & $1.6 \%$ & $0.14[0.01,2.69]$ & 2015 & $\leftarrow$ & & & & \\
\hline ESCAPE & 6 & 165 & 4 & 150 & $8.8 \%$ & $1.38[0.38,4.98]$ & 2015 & & & & & \\
\hline REVASCAT & 2 & 103 & 2 & 103 & $3.7 \%$ & $1.00[0.14,7.24]$ & 2015 & & & & & \\
\hline EXTEND-IA & 0 & 35 & 2 & 35 & $1.5 \%$ & $0.19[0.01,4.08]$ & 2015 & & & & & \\
\hline Total $(95 \% \mathrm{Cl})$ & & 1274 & & 1084 & $100.0 \%$ & $1.03[0.70,1.50]$ & & & & & & \\
\hline Total events & 65 & & 55 & & & & & & & & & \\
\hline \multirow{2}{*}{\multicolumn{8}{|c|}{$\begin{array}{l}\text { Heterogeneity: } \text { Tau }^{2}=0.00 ; \mathrm{Chi}^{2}=3.93, \mathrm{df}=7(\mathrm{P}=0.79) ; \mathrm{I}^{2}=0 \% \\
\text { Test for overall effect: } Z=0.14(P=0.89)\end{array}$}} & $0.1 \quad 0.2$ & 0.51 & 2 & 5 & 10 \\
\hline & & & & & & & & & Favours ERT & Favours Control & & \\
\hline
\end{tabular}

Figure 6. Pooled estimates for symptomatic intracranial hemorrhage (SICH) with Endovascular recanalization therapy vs. control.

trials used appropriate allocation concealment and blinded outcome assessment, and no trial contained attrition bias, reporting bias, or other forms of bias.

One critique states that only a minority of patients with AIS would be eligible for ERT but the implementation of ERT pro- grams requires tremendous resource allocation. In the USA, only 5\%-13\% of patients with AIS arrived within the commonly recommended ERT time window of 3-6 hours, and the proportion of patients eligible for ERT was estimated to be even lower. ${ }^{25}$ In Korea, about $51 \%$ of patients with AIS arrived within 6 hours 
Pooling all RCTs

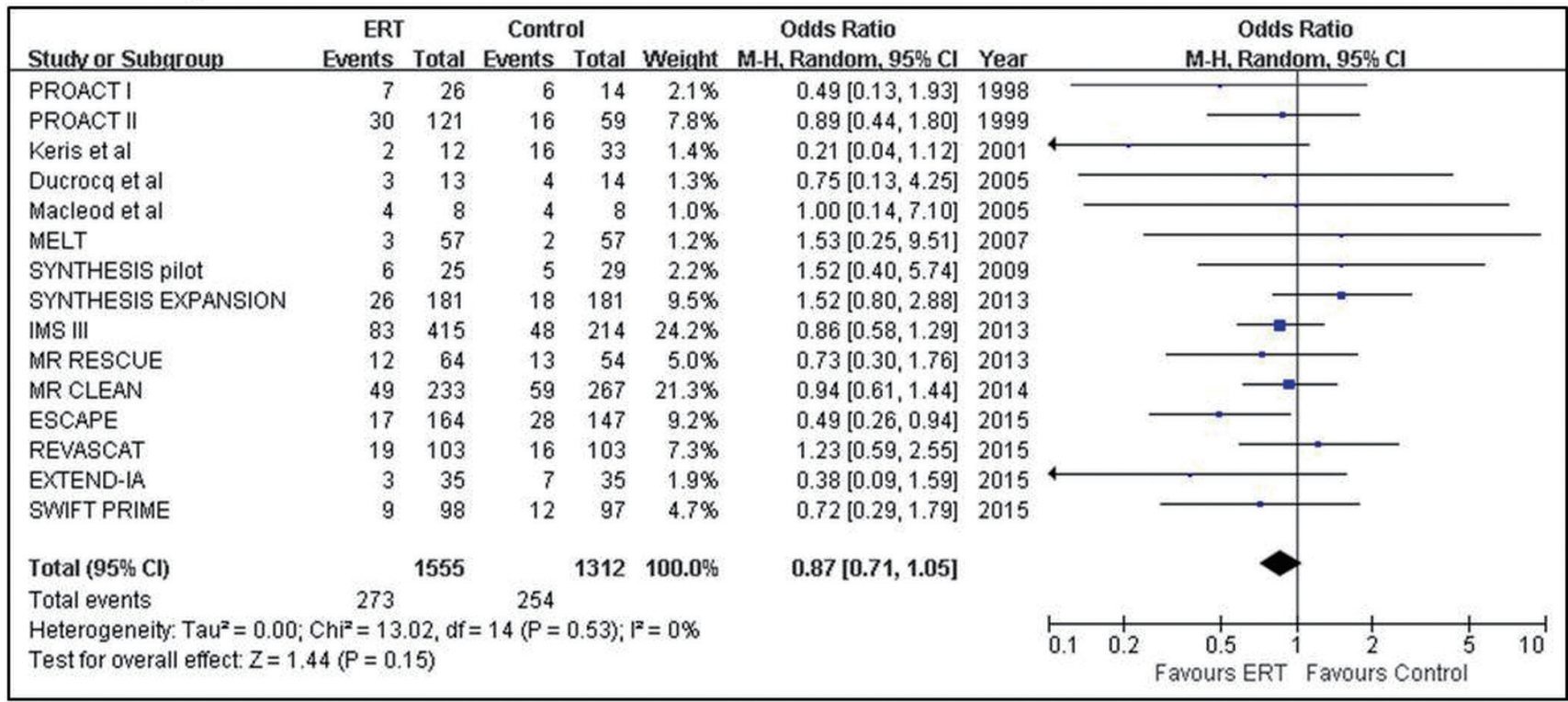

Pooling stent-retriever RCTs

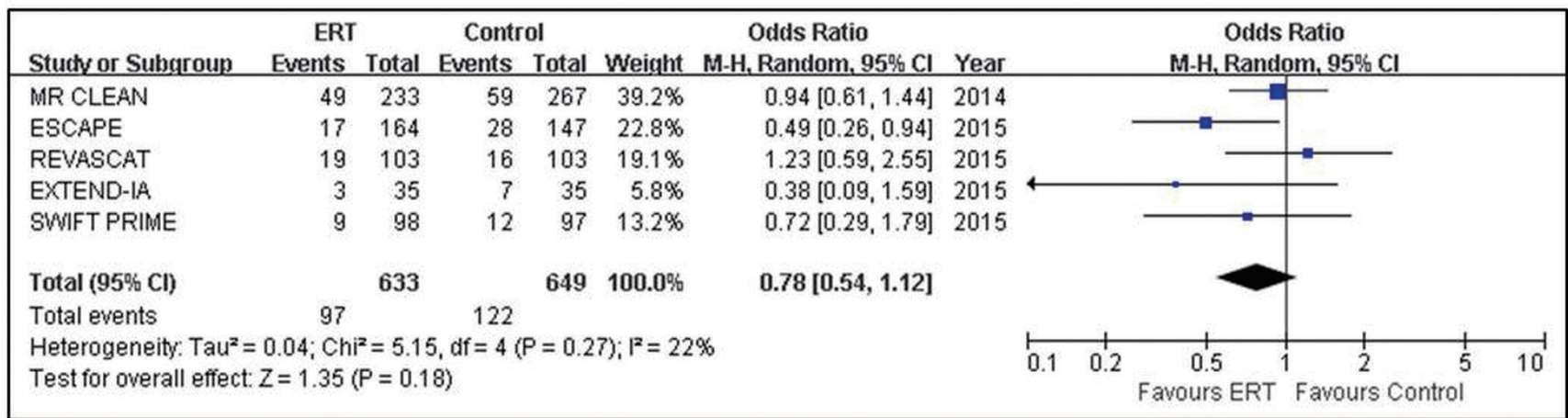

Pooling RCTs with IV-TPA rate $>75 \%$ in control arms

\begin{tabular}{|c|c|c|c|c|c|c|c|c|c|c|c|}
\hline Studve or Subqroup & $\begin{array}{l}\text { ERT } \\
\text { Events }\end{array}$ & Total & $\begin{array}{l}\text { Contr } \\
\text { Events }\end{array}$ & $\begin{array}{l}\text { ol } \\
\text { Total }\end{array}$ & Weight & $\begin{array}{c}\text { Odds Ratio } \\
\text { M-H. Random, } 95 \% \mathrm{Cl}\end{array}$ & Year & & $\begin{array}{r}\text { Odds } \mathrm{F} \\
\text { M-H. Rando }\end{array}$ & $\begin{array}{l}\text { Ratio } \\
\text { om. } 95 \% \mathrm{Cl}\end{array}$ & \\
\hline SYNTHESIS pilot & 6 & 25 & 5 & 29 & $3.7 \%$ & $1.52[0.40,5.74]$ & 2009 & & & & \\
\hline SYNTHESIS EXPANSION & 26 & 181 & 18 & 181 & $13.3 \%$ & $1.52[0.80,2.88]$ & 2013 & & & & \\
\hline IMS III & 83 & 415 & 48 & 214 & $25.2 \%$ & $0.86[0.58,1.29]$ & 2013 & & & 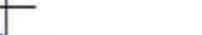 & \\
\hline MR CLEAN & 49 & 233 & 59 & 267 & $23.4 \%$ & $0.94[0.61,1.44]$ & 2014 & & & & \\
\hline ESCAPE & 17 & 164 & 28 & 147 & $13.0 \%$ & $0.49[0.26,0.94]$ & 2015 & & & & \\
\hline SWIFT PRIME & 9 & 98 & 12 & 97 & $7.4 \%$ & $0.72[0.29,1.79]$ & 2015 & & & & \\
\hline REVASCAT & 19 & 103 & 16 & 103 & $10.8 \%$ & $1.23[0.59,2.55]$ & 2015 & & & & \\
\hline EXTEND-IA & 3 & 35 & 7 & 35 & $3.2 \%$ & $0.38[0.09,1.59]$ & 2015 & $\leftarrow$ & & & \\
\hline Total $(95 \% \mathrm{Cl})$ & & 1254 & & 1073 & $100.0 \%$ & $0.90[0.69,1.17]$ & & & & & \\
\hline Total events & 212 & & 193 & & & & & & & & \\
\hline \multicolumn{8}{|c|}{$\begin{array}{l}\text { Heterogeneity: } \text { Tau }^{2}=0.03 ; \mathrm{Chi}^{2}=8.93, \mathrm{df}=7(P=0.26) ; \mathrm{I}^{2}=22 \% \\
\text { Test for overall effect: } Z=0.79(P=0.43)\end{array}$} & $0.1 \quad 0.2$ & $\begin{array}{c}0.5 \\
\text { Favours ERT }\end{array}$ & ${ }^{1}{ }_{\text {Favours Control }}^{2}$ & 10 \\
\hline
\end{tabular}

Figure 7. Pooled estimates for mortality with Endovascular recanalization therapy vs. control.

according to the Report of Assessment for Quality of Acute Stroke Care in Korea 2010. ${ }^{26}$ In a multicenter study, among 27,851 patients with AIS, 4.6\% were treated with ERT (ERT alone or combined with IV-TPA). ${ }^{27}$ However, patients with severe stroke are an important group which requires more effective interventions. In recent stent-retriever trials, only $26.4 \%$ of patients in control arms, despite the high IV-TPA rate of 77\%$100 \%$ across the trials, achieved mRS 0-2 outcomes at 3 months (Table 3). In Korea, cardioembolic stroke, which usually presents as severe stroke and might be indicated for ERT in a large 
Pooling all RCTs

\begin{tabular}{|c|c|c|c|c|c|c|c|c|c|c|c|}
\hline Stucty or Subgroup & $\begin{array}{l}\text { ERT } \\
\text { Events }\end{array}$ & Total & $\begin{array}{l}\text { Contr } \\
\text { Events }\end{array}$ & $\begin{array}{l}\text { ol } \\
\text { Total }\end{array}$ & weight & $\begin{array}{l}\text { Odds Ratio } \\
\text { M-H. Random, } 95 \% \mathrm{Cl}\end{array}$ & Year & & $\begin{array}{r}\text { Odds F } \\
\text { M-H. Rando }\end{array}$ & $\begin{array}{l}\text { Ratio } \\
\text { om. } 95 \% \mathrm{Cl}\end{array}$ & \\
\hline PROACT II & 41 & 121 & 20 & 59 & $8.6 \%$ & $1.00[0.52,1.93]$ & 1999 & & & & \\
\hline Macleod et al & 4 & 8 & 5 & 8 & $1.3 \%$ & $0.60[0.08,4.40]$ & 2005 & & & & \\
\hline MELT & 12 & 57 & 13 & 57 & $5.5 \%$ & $0.90[0.37,2.19]$ & 2007 & & & & \\
\hline SYNTHESIS pilot & 6 & 25 & 5 & 29 & $2.8 \%$ & $1.52[0.40,5.74]$ & 2009 & & & & \\
\hline SYNTHESIS EXPANSION & 36 & 181 & 31 & 181 & $11.4 \%$ & $1.20[0.71,2.04]$ & 2013 & & & & \\
\hline MR RESCUE & 27 & 64 & 19 & 54 & $7.2 \%$ & $1.34[0.64,2.84]$ & 2013 & & & & \\
\hline IMS III & 103 & 415 & 63 & 214 & $16.4 \%$ & $0.79[0.55,1.14]$ & 2013 & & & & \\
\hline MR CLEAN & 63 & 233 & 91 & 267 & $15.8 \%$ & $0.72[0.49,1.05]$ & 2014 & & & & \\
\hline SWIFT PRIME & 12 & 98 & 24 & 97 & $7.0 \%$ & $0.42[0.20,0.91]$ & 2015 & & & & \\
\hline REVASCAT & 31 & 103 & 37 & 103 & $10.2 \%$ & $0.77[0.43,1.38]$ & 2015 & & & & \\
\hline ESCAPE & 28 & 164 & 46 & 147 & $11.3 \%$ & $0.45[0.26,0.77]$ & 2015 & & & & \\
\hline EXTEND-IA & 3 & 35 & 11 & 35 & $2.6 \%$ & $0.20[0.05,0.81]$ & 2015 & & & & \\
\hline Total $(95 \% \mathrm{Cl})$ & & 1504 & & 1251 & $100.0 \%$ & $0.77[0.61,0.97]$ & & & & & \\
\hline Total events & 366 & & 365 & & & & & & & & \\
\hline \multicolumn{8}{|c|}{$\begin{array}{l}\text { Heterogeneity: } \text { Tau }^{2}=0.05 ; \mathrm{Chi}^{2}=16.46, \mathrm{df}=11(P=0.13) ;\left.\right|^{2}=33 \% \\
\text { Test for overall effect: } Z=2.25(P=0.02)\end{array}$} & $0.1 \quad 0.2$ & $\begin{array}{l}0.5 \\
\text { avours ERT }\end{array}$ & ${ }^{2}{ }^{2}{ }^{5}$ & $10^{\circ}$ \\
\hline
\end{tabular}

Pooling stent-retriever RCTs

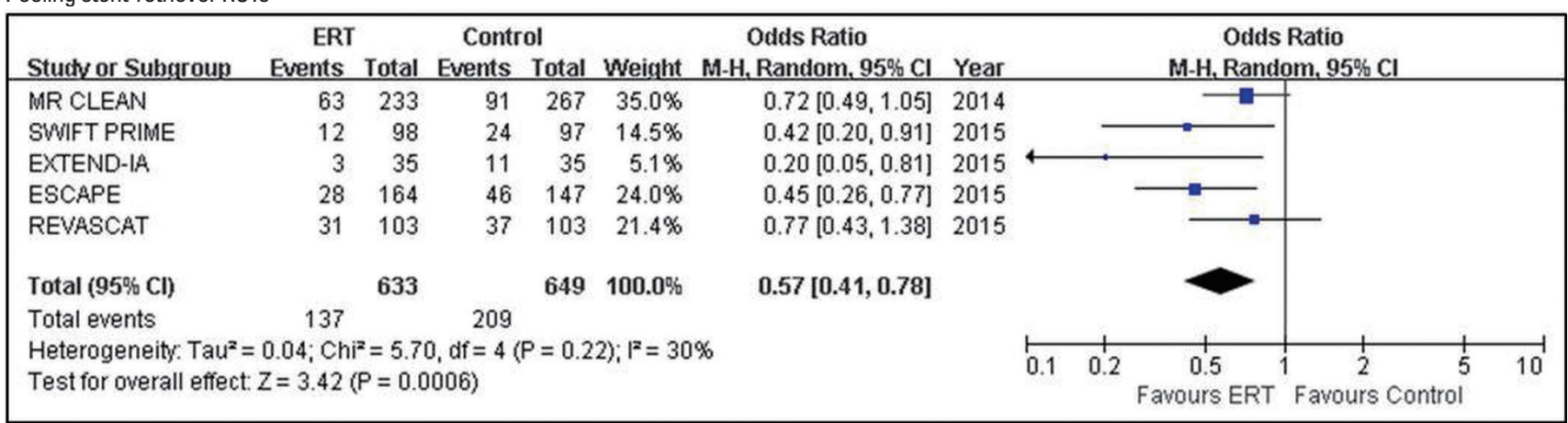

Pooling RCTs with IV-TPA rate $>75 \%$ in control arms

\begin{tabular}{|c|c|c|c|c|c|c|c|c|c|c|c|}
\hline Studve or Subqroun & $\begin{array}{l}\text { ERT } \\
\text { Events }\end{array}$ & Total & \multicolumn{2}{|c|}{ Control } & \multicolumn{2}{|c|}{$\begin{array}{c}\text { Odds Ratio } \\
\text { weight } \mathrm{M}-\mathrm{H}, \text { Random, } 95 \% \mathrm{Cl}\end{array}$} & Year & \multicolumn{3}{|c|}{$\begin{array}{l}\text { Odds Ratio } \\
\text { M-H. Random, } 95 \% \mathrm{Cl}\end{array}$} & \\
\hline SYNTHESIS pilot & 6 & 25 & 5 & 29 & $4.0 \%$ & $1.52[0.40,5.74]$ & 2009 & & & & \\
\hline SYNTHESIS EXPANSION & 36 & 181 & 31 & 181 & $14.8 \%$ & $1.20[0.71,2.04]$ & 2013 & & & & \\
\hline IMS III & 103 & 415 & 63 & 214 & $20.1 \%$ & $0.79[0.55,1.14]$ & 2013 & & & & \\
\hline MR CLEAN & 63 & 233 & 91 & 267 & $19.5 \%$ & $0.72[0.49,1.05]$ & 2014 & & & & \\
\hline EXTEND-IA & 3 & 35 & 11 & 35 & $3.8 \%$ & $0.20[0.05,0.81]$ & 2015 & $\longleftarrow$ & & & \\
\hline REVASCAT & 31 & 103 & 37 & 103 & $13.4 \%$ & $0.77[0.43,1.38]$ & 2015 & & & & \\
\hline ESCAPE & 28 & 164 & 46 & 147 & $14.7 \%$ & $0.45[0.26,0.77]$ & 2015 & & & & \\
\hline SWIFT PRIME & 12 & 98 & 24 & 97 & $9.7 \%$ & $0.42[0.20,0.91]$ & 2015 & & & & \\
\hline Total $(95 \% \mathrm{Cl})$ & & 1254 & & 1073 & $100.0 \%$ & $0.70[0.52,0.93]$ & & & & & \\
\hline Total events & 282 & & 308 & & & & & & & & \\
\hline $\begin{array}{l}\text { Heterogeneity: } \text { Tau }^{2}=0.07 \\
\text { Test for overall effect: } Z=2\end{array}$ & $\begin{array}{l}\text { Chi }=13 \\
18(P=0\end{array}$ & $\begin{array}{l}01, \mathrm{df}= \\
01)\end{array}$ & $7(P=0$ & $.07) ; 1^{2}$ & $=46 \%$ & & & $0.1 \quad 0.2$ & $\begin{array}{c}0.5 \\
\text { Favours ERT }\end{array}$ & $\begin{array}{cr}1 & 1 \\
\text { Favours Control } & 5\end{array}$ & 10 \\
\hline
\end{tabular}

Figure 8. Pooled estimates for bed-ridden or dead outcome (mRS 5-6) with Endovascular recanalization therapy vs. control.

proportion of patients arriving within time window, has increased and currently accounts for more than $20 \%$ of all AIS cases, ${ }^{28,29}$ and will further increase in the near future due to a rapid increase of elderly population. With current standard therapies, approximately one-third of patients with cardioembolic stroke are bed-ridden or die at 3 months. ${ }^{30}$ According to a multicenter registry study in Korea, about $20 \%$ of patients had a severe stroke with an initial NIHSS score of 10 or more likely caused by an acute large artery occlusion. ${ }^{27}$ Therefore, patients eligible for ERT do not represent the minority of all ischemic stroke patients.

Our study has several limitations. As with all meta-analyses, there is a risk of publication bias and of failing to identify all relevant studies. However, we included all large RCTs, and missing 
some small trials would therefore not alter our results. In addition, we could not identify publication bias for any outcome except for SICH in recent stent-retriever trials. Inspecting a funnel plot showed that bias was more likely to be driven by extremely low SICH rates in the ERT arms of two trials ${ }^{9,10}$ rather than a true significant bias. Given the variation in the definitions of SICH used across trials, the current pooled estimate of the $\mathrm{SICH}$ risk might be imprecise. However, the variation in $\mathrm{SICH}$ definition was not substantial. This study-level meta-analysis was not able to indicate the benefits of ERT in important subgroups. A pooled analysis of individual patient data from recent stent-retriever thrombectomy trials is warranted to provide more insights into the efficacy of ERT according to age, initial stroke severity, occlusion site, additional benefit or safety of prior IV-TPA use, onset-to-treatment and onset-to-recanalization times, and final reperfusion grade. Our findings were generated from data from clinical trials that generally enrolled patients at centers equipped with efficient critical pathways and experienced neurointerventionists. Therefore, these results may not be applicable to inexperienced centers.

\section{Conclusion}

In conclusion, the current updated meta-analysis shows that ERT compared to the current standard therapy substantially improves clinical outcomes and reduces extreme disability or death. Despite an increased risk of any ICH, ERT does not increase mortality or SICH. The time has come to reorganize our acute stroke care system to provide ERT for eligible patients more frequently and faster.

\section{References}

1. Tissue plasminogen activator for acute ischemic stroke. The National Institute of Neurological Disorders and Stroke rt-PA Stroke Study Group. N Engl J Med 1995;333:1581-1587.

2. Hacke W, Kaste M, Bluhmki E, Brozman M, Davalos A, Guidetti $\mathrm{D}$, et al. Thrombolysis with alteplase 3 to 4.5 hours after acute ischemic stroke. N Engl J Med 2008;359:1317-1329.

3. Riedel CH, Zimmermann P, Jensen-Kondering U, Stingele R, Deuschl G, Jansen O. The importance of size: successful recanalization by intravenous thrombolysis in acute anterior stroke depends on thrombus length. Stroke 2011;42:1775-1777.

4. Broderick JP, Palesch YY, Demchuk AM, Yeatts SD, Khatri P, Hill MD, et al. Endovascular therapy after intravenous t-PA versus t-PA alone for stroke. N Engl J Med 2013;368:893-903.

5. Lee M, Hong KS, Saver JL. Efficacy of intra-arterial fibrinolysis for acute ischemic stroke: meta-analysis of randomized con- trolled trials. Stroke 2010;41:932-937.

6. Ciccone A, Valvassori L, Nichelatti M, Sgoifo A, Ponzio M, Sterzi R, et al. Endovascular treatment for acute ischemic stroke. NEngl J Med 2013;368:904-913.

7. Berkhemer OA, Fransen PS, Beumer D, van den Berg LA, Lingsma HF, Yoo AJ, et al. A randomized trial of intraarterial treatment for acute ischemic stroke. NEngl J Med 2015;372:11-20.

8. Goyal M, Demchuk AM, Menon BK, Eesa M, Rempel JL, Thornton J, et al. Randomized assessment of rapid endovascular treatment of ischemic stroke. N Engl J Med 2015;372:1019-1030.

9. Campbell BC, Mitchell PJ, Kleinig TJ, Dewey HM, Churilov L, Yassi N, et al. Endovascular therapy for ischemic stroke with perfusion-imaging selection. NEngl J Med 2015;372:1009-1018.

10. Saver JL, Goyal M, Bonafe A, Diener HC, Levy EI, Pereira VM, et al. Stent-Retriever Thrombectomy after Intravenous t-PA vs. t-PA Alone in Stroke. N Engl J Med 2015:372:2285-2295.

11. Jovin TG, Chamorro A, Cobo E, de Miquel MA, Molina CA, Rovira A, et al. Thrombectomy within 8 Hours after Symptom Onset in Ischemic Stroke. N Engl J Med 2015:372:2296-2306.

12. Higgins JPT, Green S. Cochrane Handbook for Systematic Reviews of Interventions: West Sussex, UK: Cochrane Collaboration and John Wiley \& Sons Ltd, 2008.

13. Furlan A, Higashida R, Wechsler L, Gent M, Rowley H, Kase $\mathrm{C}$, et al. Intra-arterial prourokinase for acute ischemic stroke. The PROACT II study: a randomized controlled trial. Prolyse in Acute Cerebral Thromboembolism. JAMA 1999;282: 2003-2011.

14. del Zoppo GJ, Higashida RT, Furlan AJ, Pessin MS, Rowley HA, Gent M. PROACT: a phase II randomized trial of recombinant pro-urokinase by direct arterial delivery in acute middle cerebral artery stroke. PROACT Investigators. Prolyse in Acute Cerebral Thromboembolism. Stroke 1998;29:4-11.

15. Keris V, Rudnicka S, Vorona V, Enina G, Tilgale B, Fricbergs J. Combined intraarterial/intravenous thrombolysis for acute ischemic stroke. AJNR Am J Neuroradiol 2001;22:352-358.

16. Ducrocq X, Bracard S, Taillandier L, Anxionnat R, Lacour JC, Guillemin F, et al. Comparison of intravenous and intra-arterial urokinase thrombolysis for acute ischaemic stroke. J Neuroradiol 2005;32:26-32.

17. Macleod MR, Davis SM, Mitchell PJ, Gerraty RP, Fitt G, Hankey GJ, et al. Results of a multicentre, randomised controlled trial of intra-arterial urokinase in the treatment of acute posterior circulation ischaemic stroke. Cerebrovasc Dis 2005;20:12-17.

18. Ogawa A, Mori E, Minematsu K, Taki W, Takahashi A, Nemoto $\mathrm{S}$, et al. Randomized trial of intraarterial infusion of urokinase within 6 hours of middle cerebral artery stroke: the middle cerebral artery embolism local fibrinolytic intervention trial (MELT) Japan. Stroke 2007;38:2633-2639. 
19. Ciccone A, Valvassori L, Ponzio M, Ballabio E, Gasparotti R, Sessa $\mathrm{M}$, et al. Intra-arterial or intravenous thrombolysis for acute ischemic stroke? The SYNTHESIS pilot trial. J Neurointerv Surg 2010;2:74-79.

20. Kidwell CS, Jahan R, Gornbein J, Alger JR, Nenov V, Ajani Z, et al. A trial of imaging selection and endovascular treatment for ischemic stroke. N Engl J Med 2013;368:914-923.

21. Samsa GP, Matchar DB, Goldstein L, Bonito A, Duncan PW, Lipscomb J, et al. Utilities for major stroke: results from a survey of preferences among persons at increased risk for stroke. Am Heart J 1998; 136:703-713.

22. Hong KS, Saver JL. Quantifying the value of stroke disability outcomes: WHO global burden of disease project disability weights for each level of the modified Rankin Scale. Stroke 2009; 40:3828-3833.

23. Shuaib A, Lees KR, Lyden P, Grotta J, Davalos A, Davis SM, et al. NXY-059 for the treatment of acute ischemic stroke. N Engl JMed 2007;357:562-571.

24. Huynh T, Perron S, O’Loughlin J, Joseph L, Labrecque M, Tu JV, et al. Comparison of Primary Percutaneous Coronary Intervention and Fibrinolytic Therapy in ST-Segment-Elevation Myocardial Infarction: Bayesian Hierarchical Meta-Analyses of Randomized Controlled Trials and Observational Studies. Circulation 2009;119:3101-3109.
25. Zahuranec DB, Majersik JJ. Percentage of acute stroke patients eligible for endovascular treatment. Neurology 2012;79:S22-S25.

26. Hong KS, Bang OY, Kim JS, Heo JH, Yu KH, Bae HJ, et al. Stroke Statistics in Korea: Part II Stroke Awareness and Acute Stroke Care, A Report from the Korean Stroke Society and Clinical Research Center For Stroke. J Stroke 2013;15:67-77.

27. Kim BJ, Park JM, Kang K, Lee SJ, Ko Y, Kim JG, et al. Case characteristics, hyperacute treatment, and outcome information from the clinical research center for stroke-fifth division registry in South Korea. J Stroke 2015;17:38-53.

28. Jung KH, Lee SH, Kim BJ, Yu KH, Hong KS, Lee BC, et al. Secular trends in ischemic stroke characteristics in a rapidly developed country: results from the Korean Stroke Registry Study (secular trends in Korean stroke). Circ Cardiovasc Qual Outcomes 2012;5:327-334.

29. Kim BJ, Han MK, Park TH, Park SS, Lee KB, Lee BC, et al. Current status of acute stroke management in Korea: a report on a multicenter, comprehensive acute stroke registry. Int J Stroke 2014;9:514-518.

30. Hong KS, Lee J, Bae HJ, Lee JS, Kang DW, Yu KH, et al. Greater stroke severity predominates over all other factors for the worse outcome of cardioembolic stroke. J Stroke Cerebrovasc Dis 2013;22:e373-380. 
Pooling all RCTs

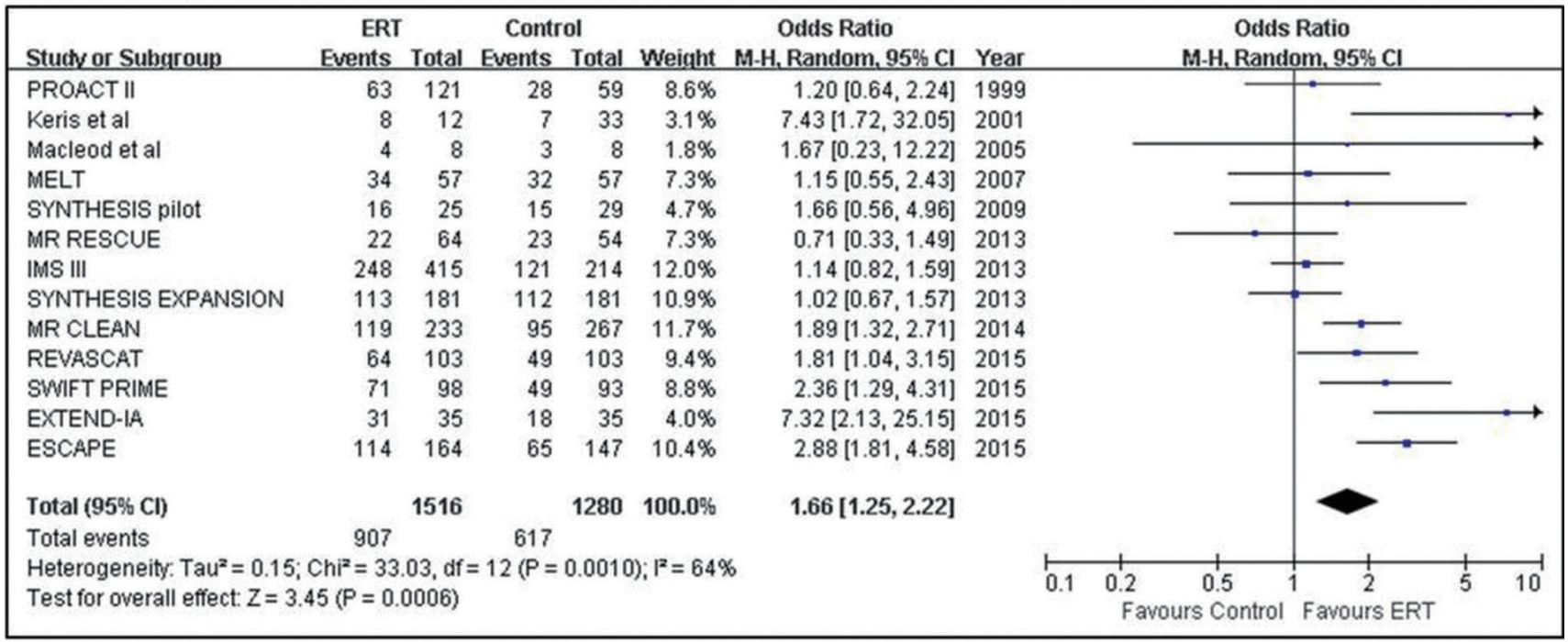

Pooling stent-retriever RCTs

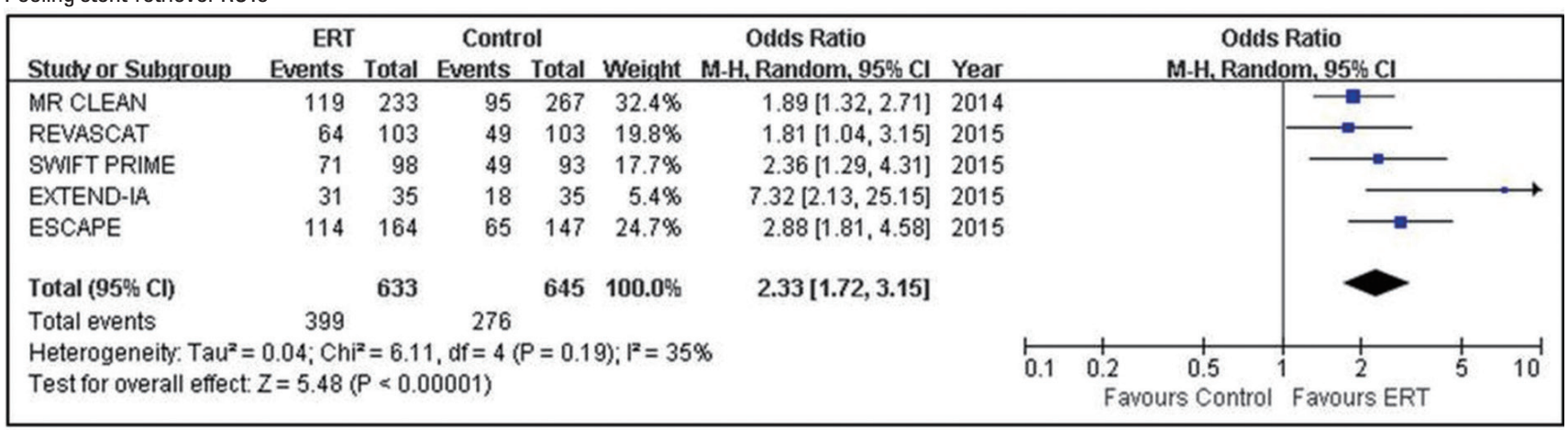

Pooling RCTs with IV-TPA rate $>75 \%$ in control arms

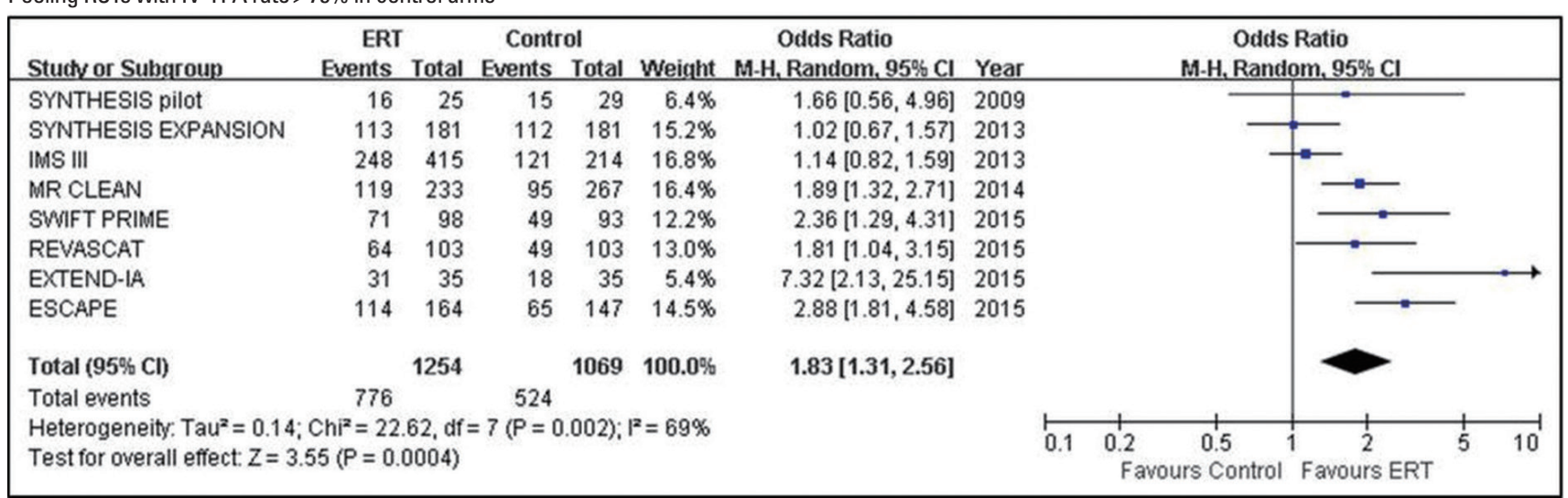

Supplementary Figure 1. Pooled estimates for achieving modified Rankin Scale (mRS) 0-3 outcomes with Endovascular recanalization therapy vs. control. 
Pooling all RCTs

\begin{tabular}{|c|c|c|c|c|c|c|c|c|c|c|c|c|}
\hline \multirow{2}{*}{$\begin{array}{l}\text { Stucty or Subgroup } \\
\text { PROACTI }\end{array}$} & \multirow{2}{*}{$\begin{array}{r}\begin{array}{r}\text { ERT } \\
\text { Events }\end{array} \\
5\end{array}$} & \multirow{2}{*}{$\frac{\text { Total }}{26}$} & $\begin{array}{l}\text { Contr } \\
\text { Events }\end{array}$ & $\begin{array}{l}\text { ol } \\
\text { Total }\end{array}$ & Weight & $\begin{array}{l}\text { Odds Ratio } \\
\text { M-H, Random, } 95 \% \mathrm{Cl}\end{array}$ & \multirow{2}{*}{$\frac{\text { Year }}{1998}$} & \multicolumn{3}{|c|}{$\begin{array}{c}\text { Odds Ratio } \\
\text { M.H, Random, } 95 \% \mathrm{Cl}\end{array}$} & & \\
\hline & & & 1 & 14 & $2.8 \%$ & $3.10[0.32,29.53]$ & & & & & & \\
\hline PROACT I & 22 & 121 & 7 & 59 & $16.8 \%$ & $1.65[0.66,4.12]$ & 1999 & & & & & \\
\hline MELT & 20 & 57 & 8 & 57 & $16.4 \%$ & $3.31[1.31,8.34]$ & 2007 & & & & & \\
\hline ESCAPE & 79 & 153 & 31 & 134 & $53.6 \%$ & $3.55[2.13,5.92]$ & 2015 & & & & & \\
\hline EXTEND-IA & 30 & 35 & 21 & 35 & $10.4 \%$ & $4.00[1.25,12.80]$ & 2015 & & & & & \\
\hline Total $(95 \% \mathrm{Cl})$ & & 392 & & 299 & $100.0 \%$ & $3.11[2.14,4.53]$ & & & & & & \\
\hline Total events & 156 & & 68 & & & & & & & & & \\
\hline $\begin{array}{l}\text { Heterogeneity. Tau } \\
\text { Test for overall effec }\end{array}$ & $\begin{array}{l}0.00 ; \mathrm{Chi}^{-} \\
\mathrm{z}=5.94(\end{array}$ & $\begin{array}{l}=2.31 \\
P<0.0\end{array}$ & $\begin{array}{l}d f=4( \\
0001)\end{array}$ & $P=0.6$ & $8) ; I^{2}=09$ & & & $0.1 \quad 0.2$ & $\begin{array}{c}0.5 \\
\text { ours Control }\end{array}$ & $\frac{1}{2}$ & 5 & 10 \\
\hline
\end{tabular}

Pooling stent-retriever RCTs

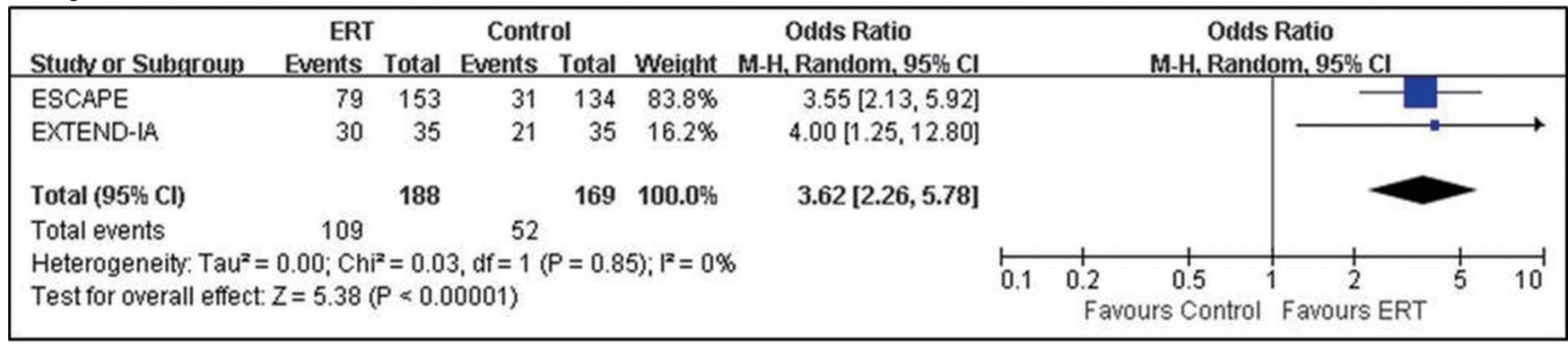

Pooling RCTs with IV-TPA rate $>75 \%$ in control arms

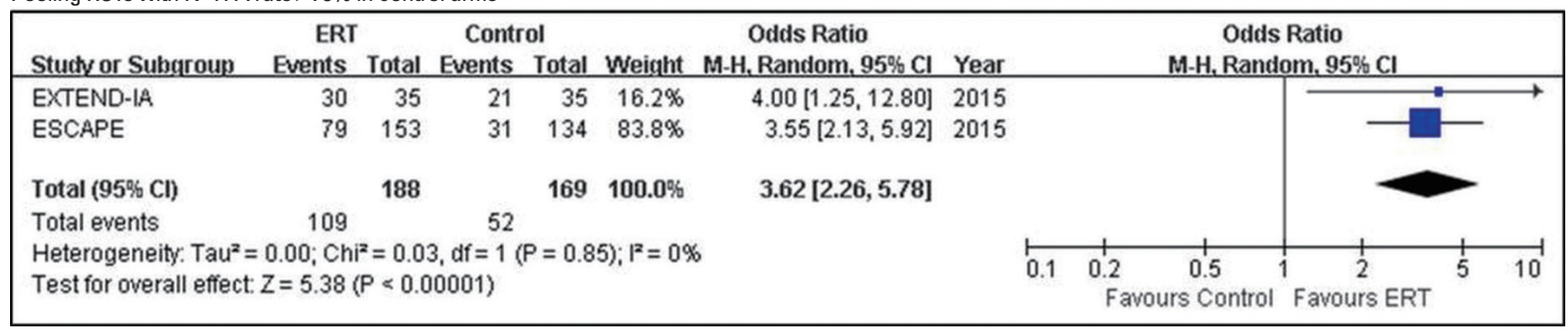


Pooling all RCTs

\begin{tabular}{|c|c|c|c|c|c|c|c|c|c|c|c|c|}
\hline Stucty or Subgroup & $\begin{array}{l}\text { ERT } \\
\text { Events }\end{array}$ & Total & $\begin{array}{l}\text { Contr } \\
\text { Events }\end{array}$ & $\begin{array}{l}\text { ol } \\
\text { Total }\end{array}$ & Weight & $\begin{array}{c}\text { Odds Ratio } \\
\text { M-H, Random, } 95 \% \mathrm{Cl}\end{array}$ & Year & & $\begin{array}{r}\text { Odds F } \\
\text { M.H. Rando }\end{array}$ & $\begin{array}{l}\text { Ratio } \\
\mathrm{om}, 95 \% \mathrm{Cl}\end{array}$ & & \\
\hline PROACTI & 11 & 26 & 5 & 14 & $3.0 \%$ & $1.32[0.35,5.05]$ & 1998 & & & & & \\
\hline PROACT ॥ & 50 & 121 & 19 & 59 & $12.4 \%$ & $1.48[0.77,2.85]$ & 1999 & & & & & \\
\hline Ducroca et al & 5 & 13 & 2 & 14 & $1.5 \%$ & $3.75[0.58,24.28]$ & 2005 & & & & & \\
\hline MELT & 28 & 57 & 19 & 57 & $9.3 \%$ & $1.93[0.91,4.12]$ & 2007 & & & & & \\
\hline MR CLEAN & 99 & 215 & 73 & 245 & $36.3 \%$ & $2.01[1.37,2.95]$ & 2014 & & & & & \\
\hline REVASCAT & 47 & 82 & 23 & 87 & $12.7 \%$ & $3.74[1.96,7.14]$ & 2015 & & & & & \\
\hline ESCAPE & 94 & 163 & 49 & 146 & $24.8 \%$ & $2.70[1.70,4.29]$ & 2015 & & & & & \\
\hline Total $(95 \% \mathrm{Cl})$ & & 677 & & 622 & $100.0 \%$ & $2.24[1.78,2.82]$ & & & & & & \\
\hline Total events & 334 & & 190 & & & & & & & & & \\
\hline \multirow{2}{*}{\multicolumn{8}{|c|}{$\begin{array}{l}\text { Heterogeneity. } \mathrm{Tau}^{2}=0.00 ; \mathrm{Ch}^{2}=5.89, \mathrm{df}=6(\mathrm{P}=0.44) ; \mathrm{I}^{2}=0 \% \\
\text { Test for overall effect } \mathrm{Z}=6.84(\mathrm{P}<0.00001)\end{array}$}} & $0.1 \quad 0.2$ & 0.51 & 2 & 5 & $\overrightarrow{10}$ \\
\hline & & & & & & & & & urs Control & Favours ERT & & \\
\hline
\end{tabular}

Pooling stent-retriever RCTs

\begin{tabular}{|c|c|c|c|c|c|c|c|c|c|c|c|c|}
\hline Stuctyor Subqroup & $\begin{array}{l}\text { ERT } \\
\text { Events }\end{array}$ & Total & $\begin{array}{l}\text { Contr } \\
\text { Events }\end{array}$ & $\begin{array}{l}\text { ol } \\
\text { Total }\end{array}$ & Weight & $\begin{array}{c}\text { Odds Ratio } \\
\text { M-H, Random, } 95 \% \mathrm{Cl}\end{array}$ & Year & & $\begin{array}{r}\text { Odds I } \\
\text { M.H. Rando }\end{array}$ & $\begin{array}{l}\text { Ratio } \\
\mathrm{om}, 95 \% \mathrm{Cl}\end{array}$ & & \\
\hline MR CLEAN & 99 & 215 & 73 & 245 & $44.4 \%$ & $2.01[1.37,2.95]$ & 2014 & & & & & \\
\hline ESCAPE & 94 & 163 & 49 & 146 & $34.7 \%$ & $2.70[1.70,4.29]$ & 2015 & & & & & \\
\hline REVASCAT & 47 & 82 & 23 & 87 & $20.9 \%$ & $3.74[1.96,7.14]$ & 2015 & & & & & \\
\hline Total $(95 \% \mathrm{Cl})$ & & 460 & & 478 & $100.0 \%$ & $2.53[1.83,3.52]$ & & & & & & \\
\hline Total events & 240 & & 145 & & & & & & & & & \\
\hline $\begin{array}{l}\text { Heterogeneity. Tau } \\
\text { Test for overall effec }\end{array}$ & $\begin{array}{l}0.02 ; \mathrm{Chi} \\
\mathrm{Z}=5.56\end{array}$ & $\begin{array}{l}=2.82 \\
P<0.0\end{array}$ & $\begin{array}{l}\text { 2. } d f=2( \\
0001)\end{array}$ & $P=0.2$ & 4); $I^{2}=29$ & & & $0.1 \quad 0.2$ & $\begin{array}{c}0.5 \\
\text { urs Control }\end{array}$ & $\frac{2}{\text { Favours EF }}$ & 5 & 10 \\
\hline
\end{tabular}

Pooling RCTs with IV-TPA rate $>75 \%$ in control arms

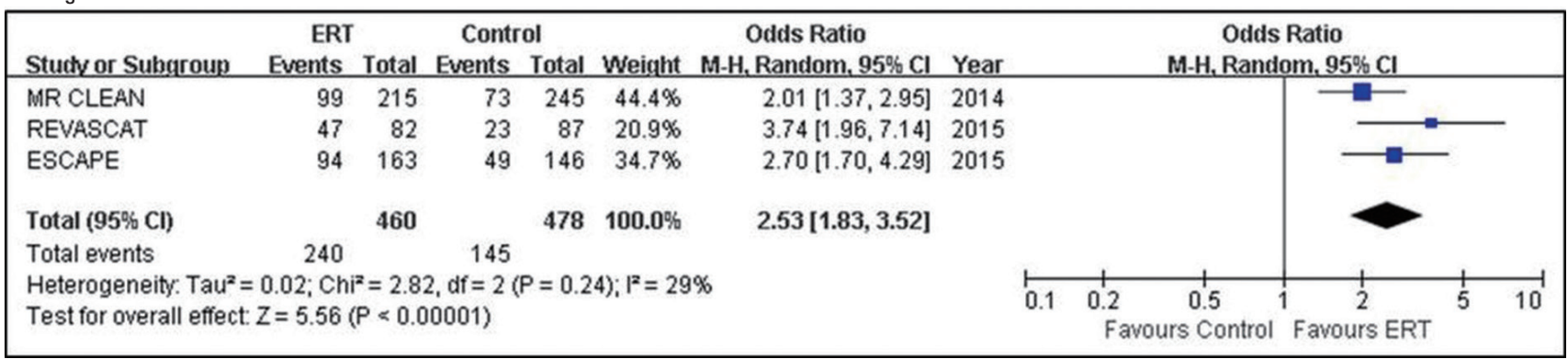

Supplementary Figure 3. Pooled estimates for achieving good activity of daily living with Endovascular recanalization therapy vs. control. 
Pooling all RCTs

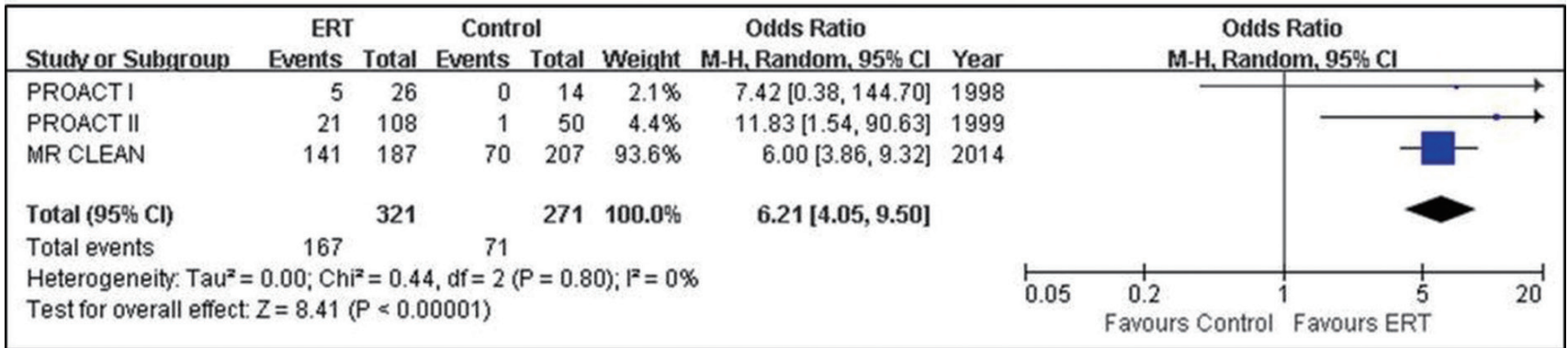

Pooling stent-retriever RCTs

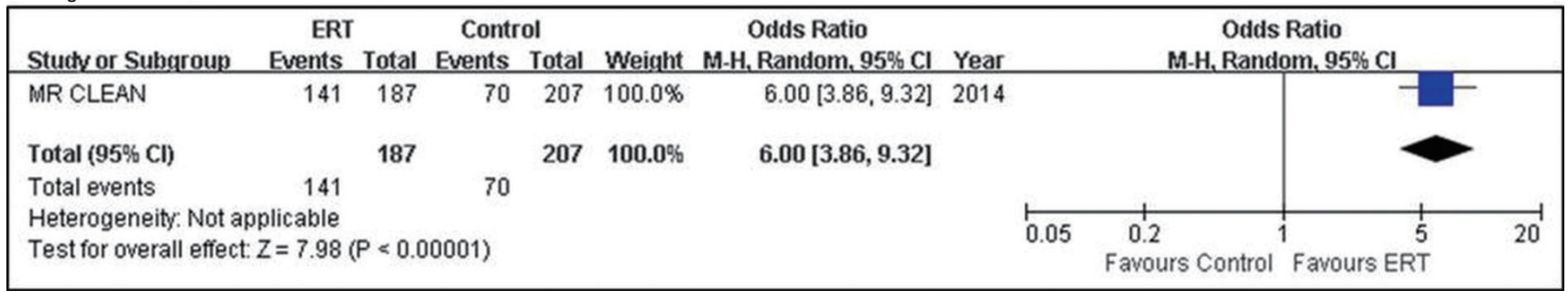

Pooling RCTs with IV-TPA rate $>75 \%$ in control arms

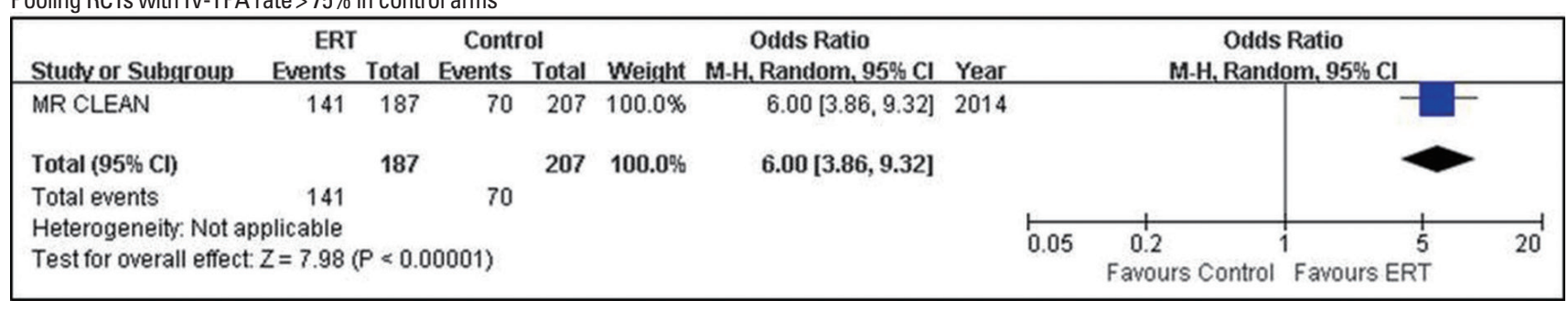

Supplementary Figure 4. Pooled estimates for achieving complete recanalization with Endovascular recanalization therapy vs. control. 
Pooling all RCTs

\begin{tabular}{|c|c|c|c|c|c|c|c|c|c|c|c|}
\hline Stuchy or Subqroup & $\begin{array}{c}\text { ERT } \\
\text { Events }\end{array}$ & Total & $\begin{array}{l}\text { Contro } \\
\text { Events }\end{array}$ & $\begin{array}{l}\text { ol } \\
\text { Total }\end{array}$ & Weight & $\begin{array}{c}\text { Odds Ratio } \\
\text { M-H, Random, } 95 \% \mathrm{Cl}\end{array}$ & Year & & $\begin{array}{r}\text { Odds } \\
\text { M-H, Rando }\end{array}$ & $\begin{array}{l}\text { Ratio } \\
\text { om, } 95 \% \mathrm{Cl}\end{array}$ & \\
\hline PROACTI & 11 & 26 & 1 & 14 & $2.3 \%$ & $9.53[1.08,84.14]$ & 1998 & & & & \\
\hline PROACTIII & 38 & 108 & 7 & 54 & $10.4 \%$ & $3.64[1.50,8.85]$ & 1999 & & & & \\
\hline Keris et al & 2 & 12 & 2 & 33 & $2.5 \%$ & $3.10[0.39,24.95]$ & 2001 & & & & \\
\hline Macleod et al & 1 & 8 & 0 & 8 & $1.0 \%$ & $3.40[0.12,96.70]$ & 2005 & & & & \\
\hline MR RESCUE & 45 & 64 & 28 & 54 & $12.8 \%$ & $2.20[1.03,4.69]$ & 2013 & & & & \\
\hline IMS III & 146 & 434 & 55 & 222 & $24.5 \%$ & $1.54[1.07,2.22]$ & 2013 & & & & \\
\hline SWIFT PRIME & 9 & 98 & 11 & 97 & $9.7 \%$ & $0.79[0.31,2.00]$ & 2015 & & & & \\
\hline REVASCAT & 19 & 103 & 13 & 103 & $12.6 \%$ & $1.57[0.73,3.37]$ & 2015 & & & & \\
\hline ESCAPE & 66 & 165 & 28 & 150 & $19.2 \%$ & $2.90[1.73,4.86]$ & 2015 & & & & \\
\hline EXTEND-IA & 4 & 35 & 5 & 35 & $5.0 \%$ & $0.77[0.19,3.16]$ & 2015 & & & & \\
\hline Total $(95 \% \mathrm{Cl})$ & & 1053 & & 770 & $100.0 \%$ & $1.93[1.37,2.72]$ & & & & & \\
\hline Total events & 341 & & 150 & & & & & & & & \\
\hline $\begin{array}{l}\text { Heterogeneity. Tau } \\
\text { Test for overall effec }\end{array}$ & $\begin{array}{l}0.09 ; \mathrm{Chi} \\
\mathrm{Z}=3.77\end{array}$ & $\begin{array}{l}z=13.7 \\
P=0.0\end{array}$ & $\begin{array}{l}8, d f=9 \\
002)\end{array}$ & $(P=0$. & $13) ; 1^{2}=35$ & $5 \%$ & & $\begin{array}{ll} \\
0.1 & 0.2\end{array}$ & $\begin{array}{c}0.5 \\
\text { avours ERT }\end{array}$ & Favours Control & 10 \\
\hline
\end{tabular}

Pooling stent-retriever RCTs

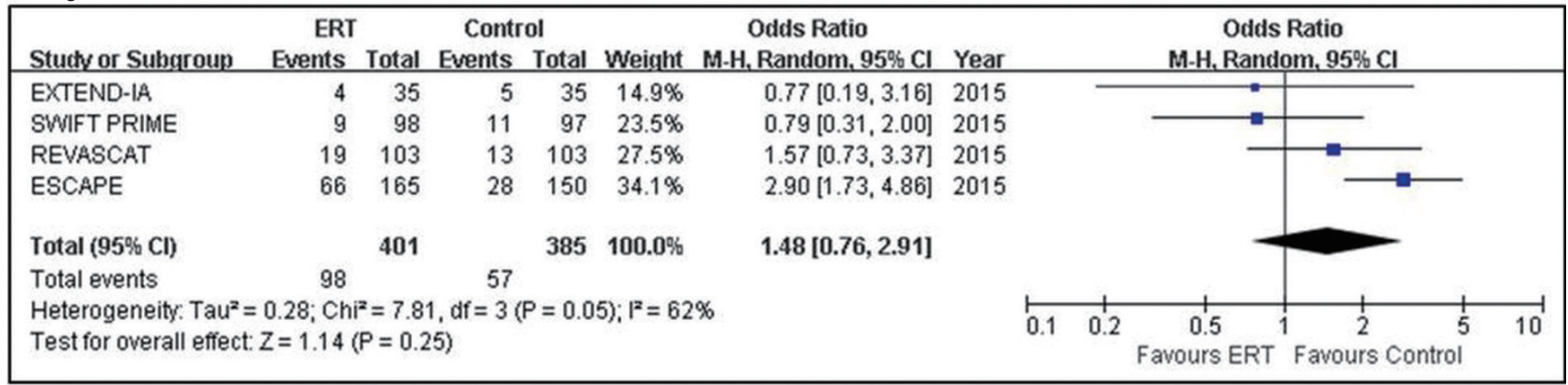

Pooling RCTs with IV-TPA rate $>75 \%$ in control arms

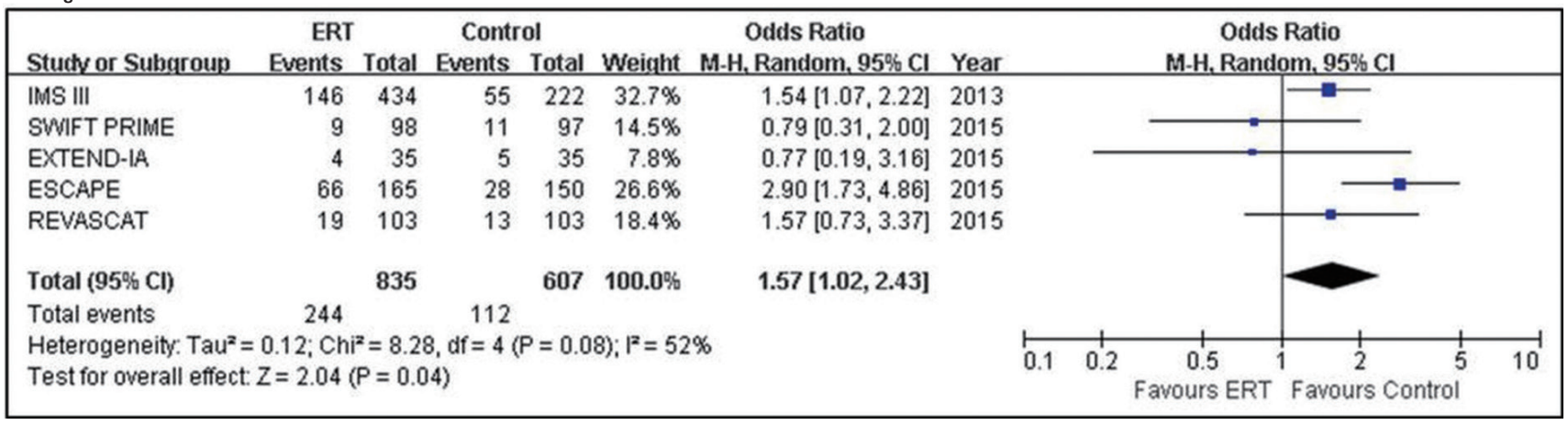

Supplementary Figure 5. Pooled estimates for any intracranial hemorrhage (ICH) with Endovascular recanalization therapy vs. control. 